\title{
SPONTANEOUS VASCULAR FLORA OF SELECTED CEMETERIES IN LUBLIN AND THE SURROUNDING AREA
}

\author{
Ewa Trzaskowska, Katarzyna Karczmarz
}

\author{
Landscaping Department, John Paul II Catholic University of Lublin \\ Konstantynów 1 H, 20-950 Lublin, Poland \\ e-mail: etrzaskowska@kul.pl, kkarczmarz@kul.pl
}

Received: 09.08.2012

\section{Abstract}

The experiment was conducted in 2011-2012. It included an analysis of the spontaneous flora of 7 cemeteries: 3 cemeteries located in the centre of Lublin (Lipowa Street, Unicka Street, and Kalina Street), 1 on the outskirts of the city (Majdanek), 1 located in a small town (Łęczna), 2 sites are rural cemeteries (Łuszczów, Ostrówek). An inventory was made of spontaneous vascular flora present at the investigated sites. The plants were described in respect of their affiliation to geographical-historical groups, life forms as well as the presence of honey species (polleniferous and nectariferous) and protected species. Within the area of these 7 necropolises, the presence of 382 taxa was found. The number of taxa observed at the individual sites varied between 124 and 274 in the metropolitan zone, 146 in the urban zone, and between 110 and 146 in the rural areas.

The area richest in terms of flora was Majdanek where 274 species of plants were found, while the cemetery in Łuszczów was the least rich -110 species. The highest precentage of hemicryptophytes was observed in Ostrówek (50.7\%), the lowest in Lublin - Kalina (40.3\%). In the case of therophytes, the greatest variation was observed in the urban cemetery in Unicka Street $(32.6 \%)$, while the least varied site was the cemetery in Ostrówek (23.3\%). The average precentage of geophytes was $12.1 \%$, with the greatest number in Łuszczów (16.4\%), and the smallest one in Majdanek (8.4\%). Chamaephytes were characterised by a small percentage (between $5.1 \%$ and $4.4 \%$ ), similarly to nanophanerophytes ( $6.2 \%$ to $2.7 \%)$, and megaphanerophytes $(9.6 \%-1.8 \%)$. The investigated sites were dominated by apophytes, the percentage of which varied between $52.3 \%$ in the Lublin cemeteries on Lipowa and Unicka Streets, and $44.5 \%$ in Ostrówek.

Among anthropophytes, there were mostly archeophytes (between $26.3 \%$ in Łuszczów and $13.1 \%$ on Lipowa St. in Lublin). The percentage of diaphytes varied between $16.5 \%$ in Ostrówek and 7.2\% in Lublin - Kalina, whereas that of kenophytes was between $15.1 \%$ in Ostrówek and 8.2\% in Łęczna. In the case of spontaneophytes, the percentage varied between $8.8 \%$ in Lublin - Majdanek and $2.7 \%$ in Łuszczów. The majo- rity, 299 of the taxa found, proved invaluable to insects (polleniferous and nectariferous). Among the plants growing spontaneously in the analysed cemeteries, there were also 11 legally protected species.

Key words: cemeteries, flora, vascular plants, cultivated species, Lublin region, Poland

\section{INTRODUCTION}

Cemeteries are considered to be green areas and cult sites that are cared for and protected ( $\mathrm{S} \mathrm{i} \mathrm{e} \mathrm{w} \mathrm{n} \mathrm{i} \mathrm{a} \mathrm{k}$ and Mitk ow ska,1998). The older ones often constitute a form of "islands" of greenery within urban and suburban development. They can present designed distribution of sites and plantings or, more often, freely growing vegetation. For many years, in Polish cemeteries there have been an increasing number of completely enclosed tombs, a new form of burial, not in earth graves, but in previously prepared brick tombs, due to which there is less space for plants. The function of burial sites dictates a specific distribution of space available for plants. Usually one can observe cut flowers in the form of wreaths, bouquets, compositions, less often vegetation planted next to the graves. Among planted plants, one can observe short forms of coniferous shrubs and individual perennial herbaceous plants.

In cities, the task of maintaining the vegetation between the sites (cleaning, mowing the lawns along the alleys, cutting trees) falls to the cemetery management. The spaces on and directly around the graves are maintained by the families of the deceased. At the same time, the specific manner of use provides diasporas of cultivated plants and synthropic species from 
outside the cemeteries. They are also transported with herbaceous material and seedlings as well as during construction and repair works ( $\mathrm{Ga}$ le $\mathrm{r}$ a et al. 1983). Therefore, in the vicinity of graves and on neglected sites one can observe greatly varied vegetation. Studies include currently used cemeteries as well as unused cemeteries ( $\mathrm{Cz}$ arn a, 2001; S o b i s z and A n t kow i a k, 2009). They concern the cemeteries of various denominations, such as, for instance, Jewish or those belonging to the Evangelical Church. Research on cemeteries relates to the dendroflora ( $\mathrm{S}$ i c i ń s k i , 1989; $\mathrm{Dąbski}$ and Oleś, 2006) and herbaceous perennial vegetation ( $\mathrm{C} \mathrm{z}$ ar n a , 2004; $\mathrm{C} \mathrm{z}$ a r n a and $\mathrm{N} \mathrm{o-}$ w iń s k a , 2011; C z a rn a et al. 2011), including ornamental plants planted purposefully in order to decorate the graves (C z e k a l s k i , 2001; D ę b i c z , 2002, 2003). A well-known work concerns grass (M o s e k and $\mathrm{Mi}$ a z g a, 2005). A multifaceted rendering and specificity of natural research of cemeteries are presented in the paper by Ję d r z j j o and W W lu s i a k (2010).

The goal of this work was to make an inventory of spontaneous flora of the cemeteries in Lublin and selected cemeteries of the Lublin region and to compare the flora in different anthropopression zones, in metropolitan, urban and rural cemeteries. There was also an attempt to ascertain the role of cemeteries as forage for insects (nectariferous and polleniferous plants) as well as the presence of protected species.

\section{MATERIALS AND METHODS}

The frequency of occurrence of spontaneous plants in cemeteries was analysed at four sites located in Lublin. Municipal and parish cemeteries (Roman-Catholic, Orthodox, or those belonging to the Evangelical Church) were included. Three of the analysed cemeteries are located in populated areas (city centre, housing estates). One (Majdanek cemetery) is located on the outskirts of the city, in the vicinity of crop fields and barren land. Another site is situated approximately $30 \mathrm{~km}$ from Lublin, in the centre of Łęczna. The two remaining sites (the cemeteries in Łuszczów and Ostrówek) are located in rural areas (Table 1).

The individual sites differ in size, which varies between 0.8 and 25 ha, the time they were established, the character of the complexes, the presence of dendroflora. The oldest of these sites is the multi-denominational cemetery on Lipowa Street; it has many trees, is under the care of a conservator, and has no space left for new graves. Groups of trees, although less numerous, are present in the older sections of the remaining cemeteries. Two of these cemeteries: Majdanek and Łęczna, can be divided into older sections and newer sections with almost no trees, and the level of insolation is high. A floristic study was conducted during 2 growing seasons in 2011-2012. Spontaneously occurring plants were included. In the case of cultivated plants, only specimens that were without doubt the effect of spontaneous renewal were taken into consideration.

A floristic analysis of the metropolitan, urban, and rural cemeteries was conducted. The qualitative composition of the flora was analysed. Using the studies by Jackowiak (1990), Zarzycki et al. (2002), and Rutkowski (2007), we were able to determine the persistence, life forms according to Raunkiaer, and affiliation to geographical-historical groups for each of the species. The nomenclature for the taxa was taken from Mirek et al. (2002) and Rutkowski (2007). The names of the ornamental plants were taken from the Zander Handwörterbuch der Pflanzenmen dictionary (Erhard t et al. 2000). Using the works by Wrzesień and Denisow (2006) as well as Lipiński (2011), honey species were also included.

Table 1

Characteristics of the examined cemeteries

in Lublin, Łęczna, Łuszczów, and Ostrówek

\begin{tabular}{lccccc}
\hline & $\begin{array}{c}\text { ATPOL } \\
\text { cemetery location }\end{array}$ & $\begin{array}{c}\text { Surface area } \\
\text { in ha }\end{array}$ & Established & Form of use & Vicinity \\
\hline Lublin - Majdanek & FE-28 & 25.0 & 1976 & in development & fields, barren land \\
Lublin - Lipowa & FE-27 & 16.6 & 1755 & no space left for new graves & urban development \\
Lublin - Unicka & FE-27 & 13.0 & 1932 & no space left for new graves & urban development \\
Lublin - Kalina & FE-27 & 3.0 & 1868 & no space left for new graves & urban development \\
Eęczna & GE-20 & 3.0 & 1955 & no space left for new graves & urban development \\
Łuszczów & FE-28 & 0.8 & 1859 & little space left for new graves & crop fields \\
Ostrówek & FD-98 & 3.7 & 1870 & little space left for new graves & crop fields \\
\hline
\end{tabular}




\section{RESULTS}

\section{Floristic abundance}

Within the area of these 7 necropolises, 382 taxa were found. The number of taxa recorded at the individual sites varied between 124 and 274 in the metropolitan zone, 146 in the urban zone, and between 110 and 146 in rural areas. The area richest in terms of flora was Majdanek where 274 species were found. The least rich in terms of species diversity is the cemetery in Euszczów, which has the smallest surface area (Table 2). In these cemeteries, one can observe a high percentage of plants spontaneously occuring near the fences, on neglected graves and between them as well as on the paths. The following are among the reasons for this high level of species diversity: duration of use, size, habitat diversity, and surroundings.

The Majdanek cemetery is the youngest and largest site which is located on the outskirts of the city among crop fields and barren land. It is dominated by insolated habitats; however, at the edge of the older section, where now there are groups of spruce and birch trees, one can observe shading. In this section,
16 species originating from forest communities were found. In the north-western section, where the sector of municipial burials with earth graves is situated, one can observe significant variation in meadow and lawn vegetation, which covers earth graves and the spaces between stone tombstones. The youngest section of the cenetery, yet not fenced in and neighbouring with crop fields, is rich in species originating from segetal communities which, in terms of phytosociology, belong to the Stellarietea mediae class.

The remaining urban cemeteries - Lipowa, Unicka, Kalina - are far smaller (3-16.6 ha) and they differ in terms of time of establishment (1755-1932) and number of species (124-176). All of them are situated in the city centre and are surrounded by development. So is the cmetery in Łęczna, which is similar in size to the one in Ostrówek. They present the same number of species; however, they differ in terms of the date of establishment and the surroundings. The smallest cemetery, in the village of Łuszczów, is one of the oldest cemeteries (1859), situated among crop fields, but also the least varied in terms of species (110).

Table 2

List of spontaneous plants found at seven cemeteries in Lublin, Łęczna, Łuszczów, and Ostrówek

\begin{tabular}{|c|c|c|c|c|c|c|c|c|c|c|c|}
\hline \multirow{2}{*}{$\begin{array}{ll} & \text { Herbaceous layer } \\
\text { Species } & \end{array}$} & \multicolumn{7}{|c|}{ Cemetery } & \multicolumn{4}{|c|}{ Species characteristics } \\
\hline & M & $\mathrm{L}$ & $\mathrm{U}$ & $\mathrm{K}$ & Łę & $€ u$ & $\mathrm{O}$ & PS & GHs & LF & $\mathrm{UF}$ \\
\hline Acer negundo L. & + & + & + & . & + & . & + & . & $\mathrm{Kn}$ & $\mathrm{M}$ & $\mathrm{P}$ \\
\hline Acer platanoides $\mathrm{L}$. & + & + & + & + & + & . & . & . & Ap & M & $\mathrm{N}, \mathrm{P}$ \\
\hline Acer pseudoplatanus $\mathrm{L}$. & + & + & . & . & . & . & . & . & Ap & M & $\mathrm{N}, \mathrm{P}$ \\
\hline Acer saccharinum $\mathrm{L}$. & + & . & . & . & . & . & . & . & $\mathrm{Kn}$ & M & $\mathrm{N}, \mathrm{P}$ \\
\hline Achillea millefolium L. s. str. & + & + & + & + & + & + & + & . & Ap & $\mathrm{G}$ & $\mathrm{N}, \mathrm{P}$ \\
\hline Achillea filipendulina Lam. & + & . & . & . & . & . & + & . & $\mathrm{Sp}$ & $\mathrm{H}$ & $\mathrm{P}$ \\
\hline Aconitum firmum Rchb. & . & . & . & & + & . & . & + & $\mathrm{D}$ & $\mathrm{H}$ & $\mathrm{N}, \mathrm{P}$ \\
\hline Aegopodium podagraria $\mathrm{L}$. & + & + & + & + & + & + & + & . & Ap & $\mathrm{H}$ & $\mathrm{N}, \mathrm{P}$ \\
\hline Aesculus hippocastanus L. & . & + & . & . & + & . & + & . & $\mathrm{D}$ & M & $\mathrm{P}$ \\
\hline Aethusa cynapium $\mathrm{L}$. & + & . & + & . & . & . & . & . & Ap & $\mathrm{T}$ & $\mathrm{N}, \mathrm{P}$. \\
\hline Agrimonia eupatoria $\mathrm{L}$. & . & . & . & . & . & . & + & . & Ap & $\mathrm{H}$ & $\mathrm{N}, \mathrm{P}$ \\
\hline Agrostis capillaris $\mathrm{L}$. & + & . & . & . & . & . & . & . & Ap & $\mathrm{H}$ & $\mathrm{P}, \mathrm{pP}$. \\
\hline Agrostis stolonifera $\mathrm{L}$. & + & + & + & + & + & . & . & . & Ap & $\mathrm{H}$ & $\mathrm{P}$ \\
\hline Ajuga reptans $\mathrm{L}$. & + & + & + & . & + & . & . & . & Ap & $\mathrm{H}$ & $\mathrm{N}, \mathrm{P}$ \\
\hline Ajuga repens ssp. purpurea & + & . & . & . & . & . & . & . & $\mathrm{Sp}$ & $\mathrm{H}$ & $\mathrm{N}, \mathrm{P}$ \\
\hline Alchemilla vulgaris $\mathrm{L}$. & . & . & . & . & . & . & + & . & $\mathrm{Sp}$ & $\mathrm{H}$ & $\mathrm{P}$ \\
\hline Alliaria petiolata (M. Bieb.) Cavara \& Grande & + & . & . & . & . & . & . & . & Ap & $\mathrm{H}$ & . \\
\hline Allium schoenoprasum $\mathrm{L}$. & . & + & + & . & + & . & . & . & $\mathrm{Kn}$ & $\mathrm{G}$ & $\mathrm{N}, \mathrm{P}$ \\
\hline Allium scorodoprasum $\mathrm{L}$. & . & . & . & . & . & + & + & . & Ap & $\mathrm{G}$ & $\mathrm{N}, \mathrm{P}$ \\
\hline Amaranthus retroflexus $\mathrm{L}$. & + & . & + & + & . & . & . & . & $\mathrm{Kn}$ & $\mathrm{T}$ & $P$ \\
\hline Anagallis arvensis $\mathrm{L}$. & + & . & + & . & + & + & . & . & $\mathrm{Ar}$ & $\mathrm{T}$ & . \\
\hline Anchusa arvensis L. & . & . & . & . & . & . & + & . & $\mathrm{Ar}$ & $\mathrm{H}$ & $\mathrm{N}, \mathrm{P}$ \\
\hline
\end{tabular}


Anchusa officinalis L.

Anemone nemorosa $\mathrm{L}$.

Anemone sylvestris L.

Anethum graveolens $\mathrm{L}$.

Angelica archangelica $\mathrm{L}$.

Anthriscus sylvestris (L.) Hoffm.

Alopecurus pratensis L.

Alopecurus geniculatus L.

Apera spica-venti (L.) P. Beauv.

Aquilegia vulgaris L.

Arabidopsis thaliana (L.) Heynh.

Arctium minus (Hill) Bernh.

Arctium tomentosum Mill.

Arenaria serpyllifolia L.

Armoracia rusticana L.

Arrhenatherum elatius (L.) P. Beauv. ex J. Presl \& C. Presl

Artemisia absintium L.

Artemisia vulgaris L.

Artemisia purshiana Bess.

Aster novi-belgii L.

Asparagus officinalis L.

Astragalus glycyphyllos L.

Athyrium filix-femina (L.) Roth.

Atriplex patula $\mathrm{L}$.

Atriplex prostrata Boucher ex DC

Atriplex hortensis L.

Atriplex hordensis rubra $\mathrm{L}$.

Avena fatua $\mathrm{L}$.

Ballota nigra $\mathrm{L}$.

Barbarea vulgaris $\mathrm{R}$. Br.

Bergenia cordifolia (Haw.) Sternb.

Bellis perennis $\mathrm{L}$.

Berteroa incana (L.) DC.

Betonica officinalis L.

Betula pubescens Ehrh.

Bidens frondosa $\mathrm{L}$.

Briza media L.

Bromus hordeaceus L.

Bromus inermis Leyss.

Bromus sterilis L.

Bromus tectorum L.

Bryonia alba $\mathrm{L}$.

Bunias orientalis L.

Calamagrostis epigejos (L.) Roth

Calamagrostis xacutiflora Overdam

Calendula officinalis L.

Calystegia sepium (L.) R. Br.

\begin{tabular}{|c|c|c|}
\hline $\mathrm{Ap}$ & $\mathrm{H}$ & $\mathrm{N}, \mathrm{P}$ \\
\hline $\mathrm{Sp}$ & $\mathrm{G}$ & $\mathrm{P}$ \\
\hline $\mathrm{Sp}$ & $\mathrm{H}$ & $\mathrm{P}$ \\
\hline $\mathrm{D}$ & $\mathrm{T}$ & $\mathrm{N}, \mathrm{P}$ \\
\hline $\mathrm{Kn}$ & $\mathrm{H}$ & \\
\hline Ap & $\mathrm{H}$ & $\mathrm{N}, \mathrm{P}$ \\
\hline Ap & $\mathrm{H}$ & $\mathrm{P}$ \\
\hline Ap & $\mathrm{H}$ & $\mathrm{P}$ \\
\hline $\mathrm{Ar}$ & $\mathrm{T}$ & . \\
\hline $\mathrm{D}$ & $\mathrm{H}$ & $\mathrm{P}$ \\
\hline Ap & $\mathrm{T}$ & . \\
\hline Ap & $\mathrm{T}$ & $\mathrm{N}, \mathrm{P}$ \\
\hline Ap & $\mathrm{T}$ & $\mathrm{N}, \mathrm{P}$ \\
\hline Ap & $\mathrm{T}$ & . \\
\hline $\mathrm{Ar}$ & $\mathrm{G}$ & . \\
\hline Ap & $\mathrm{H}$ & 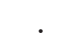 \\
\hline Ap & $\mathrm{H}$ & $\mathrm{P}$ \\
\hline Ap & $\mathrm{H}$ & $\mathrm{P}$ \\
\hline D & $\mathrm{H}$ & $\mathrm{P}$ \\
\hline $\mathrm{Kn}$ & $\mathrm{H}$ & $\mathrm{N}, \mathrm{P}$ \\
\hline $\mathrm{Kn}$ & $\mathrm{G}$ & $\mathrm{N}, \mathrm{P}$ \\
\hline Ap & $\mathrm{H}$ & $\mathrm{N}, \mathrm{P}$ \\
\hline $\mathrm{N}$ & $\mathrm{Ch}$ & $\mathrm{P}$ \\
\hline Ap & $\mathrm{T}$ & $\mathrm{P}$ \\
\hline Ap & $\mathrm{T}$ & $\mathrm{P}$ \\
\hline $\mathrm{Kn}$ & $\mathrm{T}$ & $\mathrm{P}$ \\
\hline $\mathrm{Kn}$ & $\mathrm{T}$ & $\mathrm{P}$ \\
\hline $\mathrm{Ar}$ & $\mathrm{T}$ & . \\
\hline $\mathrm{Ar}$ & $\mathrm{H}$ & $\mathrm{N}, \mathrm{P}$ \\
\hline Ap & $\mathrm{H}$ & $\mathrm{N}, \mathrm{P}$ \\
\hline $\mathrm{D}$ & $\mathrm{H}$ & $\mathrm{P}$ \\
\hline $\mathrm{Sp}$ & $\mathrm{H}$ & $\mathrm{N}, \mathrm{P}$ \\
\hline Ap & $\mathrm{T}$ & $\mathrm{N}, \mathrm{P}$ \\
\hline Ap & $\mathrm{H}$ & $\mathrm{N}, \mathrm{P}$ \\
\hline $\mathrm{Sp}$ & $\mathrm{M}$ & . \\
\hline Ap & $\mathrm{T}$ & $\mathrm{P}$ \\
\hline $\mathrm{Sp}$ & $\mathrm{H}$ & . \\
\hline Ap & $\mathrm{T}$ & \\
\hline Ap & $\mathrm{G}$ & $\mathrm{P}$ \\
\hline $\mathrm{Ar}$ & $\mathrm{T}$ & $\mathrm{P}$ \\
\hline $\mathrm{Ar}$ & $\mathrm{T}$ & $\mathrm{P}$ \\
\hline $\mathrm{Kn}$ & $\mathrm{H}$ & $\mathrm{N}, \mathrm{P}$ \\
\hline $\mathrm{Kn}$ & $\mathrm{H}$ & $\mathrm{N}, \mathrm{P}$ \\
\hline Ap & $\mathrm{G}$ & $\mathrm{P}$ \\
\hline $\mathrm{Sp}$ & $\mathrm{H}$ & $\mathrm{P}$ \\
\hline $\mathrm{D}$ & $\mathrm{Ch}$ & $\mathrm{N}, \mathrm{P}$ \\
\hline $\mathrm{Sp}$ & $\mathrm{G}$ & $\mathrm{N}, \mathrm{P}$ \\
\hline
\end{tabular}




\begin{tabular}{|c|c|c|c|c|c|c|c|c|c|c|c|}
\hline Campanula glomerata $\mathrm{L}$. & + & + & . & . & . & . & + & . & $\mathrm{Sp}$ & $\mathrm{H}$ & $\mathrm{N}, \mathrm{P}$ \\
\hline Campanula rapunculoides $\mathrm{L}$. & + & + & + & + & + & + & . & . & Ap & $\mathrm{H}$ & $\mathrm{N}, \mathrm{P}$ \\
\hline Campanula carpatica Jacq. & + & . & . & . & . & . & . & . & $\mathrm{Sp}$ & $\mathrm{H}$ & $\mathrm{N}, \mathrm{P}$ \\
\hline Capsella bursa-pastoris (L.) Medik. & + & + & + & + & + & + & + & . & $\mathrm{Ar}$ & $\mathrm{T}$ & . \\
\hline Cardamine hirsuta $\mathrm{L}$. & + & . & . & . & . & . & . & . & $\mathrm{Ar}$ & $\mathrm{T}$ & $\mathrm{N}, \mathrm{P}$ \\
\hline Carduus crispus L. & + & . & + & . & . & . & . & . & Ap & $\mathrm{H}$ & $\mathrm{N}, \mathrm{P}$ \\
\hline Carduus acanthoides $\mathrm{L}$. & . & . & + & . & . & . & . & . & $\mathrm{Ar}$ & $\mathrm{H}$ & $\mathrm{N}, \mathrm{P}$ \\
\hline Carex hirta $\mathrm{L}$. & + & + & + & . & . & . & . & . & Ap & G & . \\
\hline Carex spicata Huds. & + & + & + & + & + & . & . & . & Ap & $\mathrm{H}$ & . \\
\hline Cardaminopsis arenosa (L.) Hayek & + & . & . & . & . & . & . & . & Ap & $\mathrm{H}$ & $\mathrm{N}, \mathrm{P}$ \\
\hline Centaurea cyanus $\mathrm{L}$. & + & + & . & . & . & + & . & . & Ar & $\mathrm{T}$ & $\mathrm{N}, \mathrm{P}$ \\
\hline Centaura scabiosa $\mathrm{L}$. & + & + & . & . & . & . & + & . & Ap & $\mathrm{H}$ & $\mathrm{N}, \mathrm{P}$ \\
\hline Centaurea jacea $\mathrm{L}$. & + & . & . & . & . & . & + & . & Ap & $\mathrm{H}$ & $\mathrm{N}, \mathrm{P}$ \\
\hline Centaurea montana $\mathrm{L}$. & + & + & + & . & . & . & . & . & $\mathrm{D}$ & $\mathrm{H}$ & $\mathrm{N}, \mathrm{P}$ \\
\hline Cerastium arvense L. s. str. & + & + & + & . & . & + & . & . & Ap & $\mathrm{H}$ & $\mathrm{N}, \mathrm{P}$ \\
\hline Cerastium biebersteinii DC. & + & . & . & . & . & + & . & . & $\mathrm{D}$ & $\mathrm{H}$ & $\mathrm{N}, \mathrm{P}$ \\
\hline Cerastium glomeratum Thuill. & . & + & . & . & . & . & . & . & $\mathrm{Sp}$ & $\mathrm{H}$ & $\mathrm{N}, \mathrm{P}$ \\
\hline Cerasus avium (L.)Moench & . & + & . & . & . & . & + & . & $\mathrm{Kn}$ & M & $\mathrm{N}, \mathrm{P}$ \\
\hline Cerasus vulgaris Mill. & . & . & . & . & + & . & . & . & $\mathrm{D}$ & M & $\mathrm{N}, \mathrm{P}$ \\
\hline Chaenorhinum minus (L.) Lange & + & . & + & . & . & . & . & . & $\mathrm{Ar}$ & $\mathrm{T}$ & . \\
\hline Chaerophyllum temulum $\mathrm{L}$. & . & + & . & + & . & . & . & . & Ap & $\mathrm{H}$ & $\mathrm{P}$ \\
\hline Chamomilla suaveolens (Pursh) Rydb. & & + & + & + & + & + & + & . & $\mathrm{Kn}$ & $\mathrm{T}$ & $\mathrm{N}, \mathrm{P}$ \\
\hline Cheiranthus cheiri L. & . & . & . & . & . & . & + & . & $\mathrm{D}$ & $\mathrm{C}$ & $\mathrm{N}, \mathrm{P}$ \\
\hline Chelidonium majus L. & + & + & + & + & + & + & + & . & Ap & $\mathrm{H}$ & $\mathrm{P}$ \\
\hline Chenopodium album $\mathrm{L}$. & + & + & + & + & + & + & + & . & Ap & $\mathrm{T}$ & $\mathrm{P}$ \\
\hline Chenopodium strictum Roth & + & . & + & . & . & . & . & . & $\mathrm{Kn}$ & $\mathrm{T}$ & $\mathrm{P}$ \\
\hline Chrysanthemum indicum $\mathrm{L}$. & . & . & . & + & . & . & . & . & cul & $\mathrm{H}$ & $\mathrm{P}$ \\
\hline Cichorium intybus L. & & . & + & + & + & + & + & & $\mathrm{Ar}$ & $\mathrm{H}$ & $\mathrm{N}, \mathrm{P}$ \\
\hline Cirsium arvense (L.) Scop. & & + & + & + & + & + & + & . & Ap & G & $\mathrm{N}, \mathrm{P}$ \\
\hline Colchicum autumnale $\mathrm{L}$. & + & . & . & . & . & . & . & . & $\mathrm{Sp}$ & G & $\mathrm{N}, \mathrm{P}$ \\
\hline Consolida regalis S.F Gray. & + & . & . & . & + & + & . & . & $\mathrm{Ar}$ & $\mathrm{T}$ & $\mathrm{N}, \mathrm{P}$ \\
\hline Convallaria majalis $\mathrm{L}$. & + & + & + & + & + & + & + & . & $\mathrm{Sp}$ & G & $\mathrm{N}, \mathrm{P}$ \\
\hline Convolvulus arvensis $\mathrm{L}$. & + & + & + & + & + & + & + & . & Ap & G & $\mathrm{N}, \mathrm{P}$ \\
\hline Conyza canadensis (L.) Cronquist & + & + & + & + & + & + & + & . & $\mathrm{Kn}$ & $\mathrm{T}$ & $\mathrm{P}$ \\
\hline Cornus alba $\mathrm{L}$. & + & . & . & . & . & . & . & . & $\mathrm{Sp}$ & $\mathrm{N}$ & $\mathrm{P}$ \\
\hline Coronilla varia $\mathrm{L}$. & + & . & . & . & . & + & + & . & Ap & $\mathrm{H}$ & $\mathrm{N}, \mathrm{P}$ \\
\hline Corylus avellana $\mathrm{L}$. & + & . & . & . & . & . & . & . & Ap & $\mathrm{N}$ & $\mathrm{P}$ \\
\hline Cosmos bipinnatus & + & + & + & + & . & . & . & . & $\mathrm{D}$ & $\mathrm{T}$ & $\mathrm{P}$ \\
\hline Carpinus betulus $\mathrm{L}$. & + & + & . & . & . & . & . & . & Ap & M & $\mathrm{P}$ \\
\hline Crataegus sp. L. & + & . & . & . & . & . & . & . & Ap & $\mathrm{N}$ & $\mathrm{N}, \mathrm{P}$ \\
\hline Crepis capillaris (L.) Wallr. & + & . & + & . & . & . & . & . & $\mathrm{Sp}$ & $\mathrm{T}$ & $\mathrm{N}, \mathrm{P}$ \\
\hline Dactylis glomerata L. & + & + & + & . & + & + & . & . & Ap & $\mathrm{H}$ & . \\
\hline Daucus carota $\mathrm{L}$. & + & . & + & + & + & . & . & . & Ap & $\mathrm{T}$ & $\mathrm{N}, \mathrm{P}$ \\
\hline Delphinium elatum $\mathrm{L}$. & . & . & . & . & . & . & + & . & $\mathrm{N}$ & $\mathrm{H}$ & $\mathrm{P}$ \\
\hline Deschampsia caespitosa (L.) P. Beauv. & + & . & . & . & . & . & . & . & $\mathrm{Sp}$ & $\mathrm{H}$ & . \\
\hline Descurainia sophia $(\mathrm{L}$.$) Webb ex Prantal$ & + & + & + & + & + & + & . & . & $\mathrm{Ar}$ & $\mathrm{T}$ & $\mathrm{N}, \mathrm{P}$ \\
\hline Digitaria sanguinalis (L.)Scop. & . & . & . & + & . & . & . & . & $\mathrm{D}$ & $\mathrm{H}$ & . \\
\hline Dianthus barbatus L. s. str. & + & + & + & + & + & . & . & . & $\mathrm{Kn}$ & $\mathrm{Ch}$ & $\mathrm{N}, \mathrm{P}$ \\
\hline
\end{tabular}


Diplotaxis muralis (L.) DC.

Dipsacus sylvestris Huds.

Doronicum orientale Hoffm.

Dryopteris filix-mas (L.) Schott

Echinochloa crus-galli (L.) P. Beauv.

Echium vulgare $\mathrm{L}$.

Elymus repens (L.) Gould

Epilobium hirsutum L.

Equisetum arvense $\mathrm{L}$.

Eragrostis minor Host

Erigeron annuus (L.) Pers.

Erigeron acer L. Poggenb.

Eryngium campestre L.

Erodium cicutarium (L.) L' Her.

Erophila verna (L.) Chevall.

Erysimum cheiranthoides L.

Euonymus europaea L.

Euphorbia cyparissias L.

Euphorbia esula L.

Euphorbia marginata Pursh

Euphorbia peplus L.

Euphorbia helioscopia L.

Euphorbia humifusa Willd.

Exacum affine Balf.f. ex Regel

Falcaria vulgaris Bernh.

Fallopia convolvulus (L.) A. Love

Ficaria verna Huds.

Forsythia $x$ intermedia Zabel

Fragaria vesca $\mathrm{L}$.

Fragaria $x$ ananassa (Duchesne ex Rozier)

Fraxinus excelsior L.

Fumaria schleicheri Soy.-Will.

Gaillardia aristata Pursh

Gaillardia x glandiflora ex van Houtte

Galeobdolon luteum Huds.

Galeopsis tetrahit $\mathrm{L}$.

Galinsoga ciliata (Raf.) S. F. Blade

Galinsoga parviflora Cav.

Goniolimon tataricum (L.) Mill

Galium aparine L.

Galium mollugo L. s. str.

Galium verum $\mathrm{L}$.

Geranium pusillum Burm. f. ex L.

Geranium robertianum L.

Geranium platypetalum L.

Geranium pratense L.

Geranium pyrenaicum Burm.fil.

Geum urbanum L.

\begin{tabular}{|c|c|c|c|c|c|c|c|c|c|c|}
\hline+ & & + & & 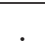 & 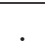 & & & $\mathrm{Kn}$ & $\mathrm{T}$ & \\
\hline+ & . & + & . & . & . & . & . & $\mathrm{Kn}$ & $\mathrm{T}$ & $\mathrm{N}, \mathrm{P}$ \\
\hline . & 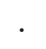 & $\cdot$ & $\cdot$ & . & . & + & . & D & $\mathrm{G}$ & $\mathrm{P}$ \\
\hline$\cdot$ & + & . & + & . & . & . & . & $\mathrm{Sp}$ & $\mathrm{H}$ & . \\
\hline+ & + & + & + & + & + & + & . & $\mathrm{Ar}$ & $\mathrm{T}$ & . \\
\hline+ & . & + & . & . & . & + & . & Ap & $\mathrm{T}$ & $\mathrm{N}, \mathrm{P}$ \\
\hline+ & + & + & + & + & + & + & . & Ap & $\mathrm{G}$ & $\mathrm{N}, \mathrm{P}$ \\
\hline+ & + & + & + & + & . & . & . & Ap & $\mathrm{H}$ & $\mathrm{N}, \mathrm{P}$ \\
\hline+ & + & + & . & + & + & + & . & Ap & $\mathrm{G}$ & . \\
\hline+ & . & . & . & . & . & . & . & $\mathrm{Kn}$ & $\mathrm{T}$ & . \\
\hline+ & + & + & + & + & + & + & . & $\mathrm{Kn}$ & $\mathrm{T}$ & $\mathrm{N}, \mathrm{P}$ \\
\hline+ & . & . & . & . & . & . & . & $\mathrm{Kn}$ & $\mathrm{T}$ & $\mathrm{N}, \mathrm{P}$ \\
\hline+ & . & + & . & . & + & + & . & $\mathrm{D}$ & $\mathrm{H}$ & $\mathrm{P}$ \\
\hline . & . & . & . & + & + & + & . & Ap & $\mathrm{T}$ & $\mathrm{N}, \mathrm{P}$ \\
\hline+ & . & . & . & . & . & . & . & Ap & $\mathrm{T}$ & . \\
\hline+ & + & + & + & + & . & . & . & Ap & $\mathrm{T}$ & . \\
\hline+ & . & . & . & . & . & . & . & Ap & $\mathrm{N}$ & $\mathrm{N}, \mathrm{P}$ \\
\hline+ & . & + & + & + & . & + & . & Ap & $\mathrm{H}$ & $\mathrm{N}, \mathrm{P}$ \\
\hline+ & . & + & . & + & . & . & . & Ap & $\mathrm{H}$ & $\mathrm{N}, \mathrm{P}$ \\
\hline$\cdot$ & . & + & . & . & . & + & . & $\mathrm{Kn}$ & $\mathrm{T}$ & $\mathrm{N}, \mathrm{P}$ \\
\hline+ & . & + & + & + & . & . & . & $\mathrm{Ar}$ & $\mathrm{T}$ & $\mathrm{N}, \mathrm{P}$ \\
\hline+ & + & + & . & . & . & . & . & $\mathrm{Ar}$ & & $\mathrm{N}, \mathrm{P}$ \\
\hline+ & . & . & . & . & . & . & . & $\mathrm{Ef}$ & $\mathrm{T}$ & $\mathrm{N}, \mathrm{P}$ \\
\hline$\cdot$ & . & . & 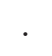 & . & . & + & . & cul & $\mathrm{T}$ & . \\
\hline . & . & . & + & . & . & . & . & Ap & $\mathrm{H}$ & $\mathrm{N}, \mathrm{P}$ \\
\hline+ & + & + & + & + & + & + & . & $\mathrm{Ar}$ & $\mathrm{T}$ & $\mathrm{N}, \mathrm{P}$ \\
\hline+ & . & 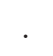 & . & . & . & + & . & $\mathrm{Sp}$ & $\mathrm{G}$ & $\mathrm{P}$ \\
\hline+ & . & + & . & . & . & . & . & $\mathrm{D}$ & $\mathrm{N}$ & $\mathrm{P}$ \\
\hline+ & . & . & 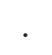 & . & . & . & . & $\mathrm{D}$ & $\mathrm{H}$ & $\mathrm{N}, \mathrm{P}$ \\
\hline . & . & + & . & . & . & . & . & $\mathrm{D}$ & $\mathrm{H}$ & $\mathrm{N}, \mathrm{P}$ \\
\hline . & + & + & + & + & . & . & . & Ap & $\mathrm{M}$ & $\mathrm{P}$ \\
\hline . & . & . & . & + & + & + & . & $\mathrm{Ar}$ & $\mathrm{T}$ & . \\
\hline+ & . & . & . & . & . & . & . & $\mathrm{D}$ & $\mathrm{H}$ & $\mathrm{P}$ \\
\hline$\cdot$ & . & + & . & . & . & . & . & $\mathrm{D}$ & $\mathrm{H}$ & $\mathrm{P}$ \\
\hline+ & . & . & . & . & . & . & . & $\mathrm{Sp}$ & $\mathrm{Ch}$ & $\mathrm{N}, \mathrm{P}$ \\
\hline+ & . & + & + & + & . & + & . & Ap & $\mathrm{T}$ & $\mathrm{N}, \mathrm{P}$ \\
\hline+ & . & . & . & . & . & . & . & $\mathrm{Kn}$ & $\mathrm{T}$ & $\mathrm{N}, \mathrm{P}$ \\
\hline+ & + & + & + & + & + & + & . & $\mathrm{Kn}$ & $\mathrm{T}$ & . \\
\hline . & . & + & . & . & . & . & . & $\mathrm{D}$ & $\mathrm{H}$ & $\mathrm{P}$ \\
\hline+ & . & . & 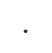 & + & . & + & . & Ap & $\mathrm{T}$ & $\mathrm{N}, \mathrm{P}$ \\
\hline+ & . & + & + & . & + & . & . & Ap & $\mathrm{H}$ & $\mathrm{N}, \mathrm{P}$ \\
\hline+ & . & . & . & . & . & . & . & Ap & $\mathrm{H}$ & $\mathrm{N}, \mathrm{P}$ \\
\hline+ & + & + & + & . & + & + & & $\mathrm{Ar}$ & $\mathrm{T}$ & $\mathrm{N}, \mathrm{P}$ \\
\hline$\cdot$ & + & $\cdot$ & $\cdot$ & . & . & . & . & $\mathrm{Sp}$ & $\mathrm{T}$ & $\mathrm{N}, \mathrm{P}$ \\
\hline$\cdot$ & $\cdot$ & . & . & . & . & + & & Ap & $\mathrm{H}$ & $\mathrm{N}, \mathrm{P}$ \\
\hline+ & + & + & + & + & . & + & . & Ap & $\mathrm{H}$ & $\mathrm{N}, \mathrm{P}$ \\
\hline+ & $\cdot$ & . & . & . & . & . & & $\mathrm{Kn}$ & $\mathrm{H}$ & $\mathrm{N}, \mathrm{P}$ \\
\hline+ & + & + & + & + & . & . & . & Ap & $\mathrm{H}$ & $\mathrm{N}, \mathrm{P}$ \\
\hline
\end{tabular}




\begin{tabular}{|c|c|c|c|c|c|c|c|c|c|c|c|}
\hline Gladiolus hybridus hort. & + & . & . & . & . & . & . & . & $\mathrm{N}$ & $\mathrm{G}$ & $\mathrm{P}$ \\
\hline Glechoma hederacea $\mathrm{L}$. & + & + & + & . & . & + & + & . & Ap & $\mathrm{H}$ & $\mathrm{N}, \mathrm{P}$ \\
\hline Gnaphalium uliginosum $\mathrm{L}$. & . & . & . & . & + & . & . & . & Ap & $\mathrm{T}$ & . \\
\hline Gypsophila paniculata $\mathrm{L}$. & . & . & . & . & . & . & + & + & $\mathrm{Sp}$ & $\mathrm{H}$ & $\mathrm{P}$ \\
\hline Hacquetia epipactis Neck.ex DC & . & . & . & . & . & . & + & . & $\mathrm{Sp}$ & $\mathrm{H}$ & . \\
\hline Hedera helix $\mathrm{L}$. & . & + & . & . & . & . & . & + & Ap & $\mathrm{Ch}$ & $\mathrm{P}$ \\
\hline Helenium autumnale $\mathrm{L}$. & + & . & . & . & . & . & + & . & $\mathrm{D}$ & $\mathrm{H}$ & . \\
\hline Helianthus rigidus (Cass.)Desf. & + & . & . & . & . & + & + & . & $\mathrm{Kn}$ & $\mathrm{G}$ & $\mathrm{N}, \mathrm{P}$ \\
\hline Heliopsis helianthoides (L.) Sweet & + & . & . & + & . & + & + & . & cul & $\mathrm{T}$ & $\mathrm{P}$ \\
\hline Hemerocallis fulva $\mathrm{L}$. & + & + & + & + & + & + & + & . & $\mathrm{D}$ & $\mathrm{H}$ & $\mathrm{P}$ \\
\hline Heracleum sphondylium $\mathrm{L}$. & + & . & . & . & + & + & + & . & Ap & $\mathrm{H}$ & $\mathrm{N}, \mathrm{P}$ \\
\hline Hesperis matronalis $\mathrm{L}$. & . & . & + & . & . & . & . & . & $\mathrm{D}$ & $\mathrm{H}$ & . \\
\hline Hieracium pilosella $\mathrm{L}$. & + & + & + & . & . & . & . & . & Ap & $\mathrm{H}$ & $\mathrm{N}, \mathrm{P}$ \\
\hline Hieracium sabaudum L. & . & + & . & . & . & . & . & . & $\mathrm{Sp}$ & $\mathrm{H}$ & $\mathrm{N}, \mathrm{P}$ \\
\hline Holcus mollis L. & . & . & . & . & + & . & . & . & Ap & $\mathrm{G}$ & . \\
\hline Holosteum umbellatum $\mathrm{L}$. & + & . & . & + & . & . & . & . & Ap & $\mathrm{T}$ & . \\
\hline Hordeum murinum $\mathrm{L}$. & + & . & + & + & & . & . & & $\mathrm{Ar}$ & $\mathrm{T}$ & . \\
\hline Humulus lupulus $\mathrm{L}$. & . & . & . & + & . & . & . & . & $\mathrm{Sp}$ & $\mathrm{H}$ & $\mathrm{N}, \mathrm{P}$ \\
\hline Hypericum perforatum $\mathrm{L}$. & + & . & + & . & + & + & + & . & Ap & $\mathrm{H}$ & $\mathrm{P}$ \\
\hline Hypochoeris radicata $\mathrm{L}$. & + & . & . & . & . & . & . & . & Ap & $\mathrm{H}$ & . \\
\hline Impatiens parviflora $\mathrm{DC}$. & + & + & + & + & + & + & + & . & $\mathrm{Kn}$ & $\mathrm{T}$ & $\mathrm{N}, \mathrm{P}$ \\
\hline Impatiens walleriana Hook.f. & + & + & . & . & + & . & . & . & cul & $\mathrm{T}$ & $\mathrm{N}, \mathrm{P}$ \\
\hline Iris germanica $\mathrm{L}$. & + & . & . & . & . & + & + & . & $\mathrm{Kn}$ & $\mathrm{H}$ & $\mathrm{N}, \mathrm{P}$ \\
\hline Juglans regia $\mathrm{L}$. & + & + & + & . & + & + & . & . & $\mathrm{D}$ & M & $\mathrm{P}$ \\
\hline Juncus tenuis Willd. & . & + & . & . & . & . & . & . & $\mathrm{Kn}$ & $\mathrm{H}$ & $\mathrm{P}$ \\
\hline Juniperus chinensis L. & . & + & . & . & . & . & . & . & $\mathrm{D}$ & $\mathrm{N}$ & . \\
\hline Knautia arvensis (L.) J.M. Coult. & . & . & . & . & . & . & + & . & Ap & $\mathrm{H}$ & $\mathrm{N}, \mathrm{P}$ \\
\hline Lactuca serriola $\mathrm{L}$. & + & + & + & + & + & . & . & . & $\mathrm{Ar}$ & $\mathrm{T}$ & $\mathrm{N}, \mathrm{P}$ \\
\hline Lamium album $\mathrm{L}$. & + & + & + & + & + & + & + & . & $\mathrm{Ar}$ & $\mathrm{H}$ & $\mathrm{N}, \mathrm{P}$ \\
\hline Lamium amplexicaule $\mathrm{L}$. & + & . & . & . & . & . & . & . & $\mathrm{Ar}$ & $\mathrm{T}$ & $\mathrm{N}, \mathrm{P}$ \\
\hline Lamium purpureum $\mathrm{L}$. & + & . & + & . & . & . & . & . & $\mathrm{Ar}$ & $\mathrm{T}$ & $\mathrm{N}, \mathrm{P}$ \\
\hline Lapsana communis L. s. str. & + & + & + & + & + & . & . & . & Ap & $\mathrm{T}$ & $\mathrm{N}, \mathrm{P}$ \\
\hline Lathyrus pratensis $\mathrm{L}$. & + & . & + & . & . & . & . & . & Ap & $\mathrm{H}$ & $\mathrm{N}, \mathrm{P}$ \\
\hline Lathyrus latifolius L. & + & . & . & . & . & . & . & + & $\mathrm{Ar}$ & $\mathrm{H}$ & $\mathrm{N}, \mathrm{P}$ \\
\hline Lathyrus tuberosus L. & + & . & . & . & . & . & . & . & $\mathrm{Ar}$ & $\mathrm{H}$ & $\mathrm{N}, \mathrm{P}$ \\
\hline Leontodon autumnalis $\mathrm{L}$. & . & . & + & . & + & . & . & . & Ap & $\mathrm{H}$ & $\mathrm{N}, \mathrm{P}$ \\
\hline Leonurus cardiaca L. & . & . & . & . & . & + & . & . & $\mathrm{Ar}$ & $\mathrm{H}$ & $\mathrm{N}, \mathrm{P}$ \\
\hline Lepidum densiflorum Schrader & + & . & . & . & . & . & . & . & $\mathrm{Kn}$ & $\mathrm{T}$ & $\mathrm{N}, \mathrm{P}$ \\
\hline Lepidum ruderale $\mathrm{L}$. & + & . & + & + & + & + & . & . & $\mathrm{Ar}$ & $\mathrm{T}$ & $\mathrm{N}, \mathrm{P}$ \\
\hline Leucanthemum vulgare Lam. s. str. & + & + & + & + & + & + & . & . & Ap & $\mathrm{H}$ & $\mathrm{N}, \mathrm{P}$ \\
\hline Ligularia dentata (A. Gray.)H. Hara & . & . & . & . & . & . & + & . & $\mathrm{D}$ & $\mathrm{H}$ & $\mathrm{P}$ \\
\hline Lilium bulbiferum $\mathrm{L}$. & . & . & . & . & . & + & . & . & $\mathrm{Sp}$ & $\mathrm{G}$ & $\mathrm{N}, \mathrm{P}$ \\
\hline Lilium candidum $\mathrm{L}$. & . & . & . & . & . & . & + & . & $\mathrm{D}$ & $\mathrm{G}$ & $\mathrm{N}, \mathrm{P}$ \\
\hline Lithospermum arvensis $\mathrm{L}$. & . & . & + & . & . & . & . & . & $\mathrm{Ar}$ & $\mathrm{T}$ & $\mathrm{N}, \mathrm{P}$ \\
\hline Lolium perenne $\mathrm{L}$. & + & + & + & + & + & + & + & . & Ap & $\mathrm{H}$ & $\mathrm{P}$ \\
\hline Lolium multiflorum (Lam.) & . & + & . & . & . & . & . & . & $\mathrm{Kn}$ & $\mathrm{H}$ & $\mathrm{P}$ \\
\hline Lotus corniculatus L. & + & . & + & . & . & . & + & . & Ap & $\mathrm{T}$ & $\mathrm{N}, \mathrm{P}$ \\
\hline Lunaria annua $\mathrm{L}$. & & & . & & . & . & + & & $\mathrm{D}$ & $\mathrm{T}$ & $\mathrm{N}, \mathrm{P}$ \\
\hline
\end{tabular}


Lupinus polyphyllus Lindl.

Lysimachia nummularia L.

Lysimachia punctata $\mathrm{L}$.

Mahonia aquifolium (Pursh)Nutt.

Malva rosea $\mathrm{L}$.

Malva neglecta L.

Malva sylvestris $\mathrm{L}$.

Matricaria maritima subsp. inodora (L.) Dostal

Matteucia struthiopteris (L.) Tod.

Medicago lupulina L.

Medicago x varia Martyn

Medicago falcata $\mathrm{L}$.

Melandrium album (Mili.) Garcke

Melilotus albus L.

Melilotus officinalis (L.) Pali.

Mentha arvensis $\mathrm{L}$.

Milium effusum L.

Morus alba L.

Muscari botryoides L.

Mycelis muralis (L.) Dumort.

Myosotis arvensis (L.) Hill

Myosotis sylvatica Ehrh. ex Hoffm.

Narcissus poeticus L.

Nepeta cataria L.

Nigella damascena $\mathrm{L}$.

Oenothera biennis L.

Oenothera missouriensis Sims

Oenothera tetragona Roth

Ornithogalum umbellatum $\mathrm{L}$.

Oxalis corniculata L.

Oxalis dillenii Jacq.

Padus serotina (Ehrh.)Borkh.

Paeonia lactiflora Pall.

Papaver argemone $\mathrm{L}$.

Papaver rhoeas L.

Papaver somniferum L.

Parthenocissus tricuspidata (Siebold \& Zucc.)

Planch. in A. \& C. DC.

Pastinaca sativa L. s. str.

Petunia $x$ atkinsiana D. Don

Philadelphus coronarius L.

Phleum pratense $\mathrm{L}$.

Phlox paniculata $\mathrm{L}$.

Phlox subulata L.

Physalis alkekengi L.

Physocarpus opulifolius (Rydb. ex Small)

Picea abies (L.) H. Karst.

Picris hieracioides L.

Pimpinella saxifraga $\mathrm{L}$.

Plantago lanceolata $\mathrm{L}$.

\begin{tabular}{|c|c|c|c|c|c|c|c|c|c|c|}
\hline+ & + & & & + & + & + & & $\mathrm{Kn}$ & $\mathrm{H}$ & $\mathrm{P}$ \\
\hline$t$ & + & + & 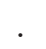 & + & $\cdot$ & + & & $\mathrm{Sp}$ & $\mathrm{H}$ & $\mathrm{N}, \mathrm{P}$ \\
\hline+ & + & + & + & + & . & . & . & $\mathrm{Sp}$ & $\mathrm{H}$ & $\mathrm{N}, \mathrm{P}$ \\
\hline+ & + & + & . & . & . & . & . & $\mathrm{Kn}$ & $\mathrm{N}$ & $\mathrm{P}$ \\
\hline+ & . & + & + & . & . & + & . & $\mathrm{D}$ & $\mathrm{H}$ & $\mathrm{N}, \mathrm{P}$ \\
\hline & . & . & . & . & . & + & . & $\mathrm{Ar}$ & $\mathrm{T}$ & $\mathrm{N}, \mathrm{P}$ \\
\hline+ & . & . & . & . & . & . & . & $\mathrm{Ar}$ & $\mathrm{T}$ & $\mathrm{N}, \mathrm{P}$ \\
\hline & . & . & . & . & . & + & . & $\mathrm{Ar}$ & $\mathrm{T}$ & $\mathrm{N}, \mathrm{P}$ \\
\hline+ & + & . & . & . & . & . & + & $\mathrm{Sp}$ & $\mathrm{H}$ & . \\
\hline+ & + & + & . & + & + & + & . & Ap & $\mathrm{H}$ & $\mathrm{N}, \mathrm{P}$ \\
\hline+ & . & + & . & . & + & . & . & $\mathrm{Kn}$ & $\mathrm{H}$ & $\mathrm{N}, \mathrm{P}$ \\
\hline+ & . & + & . & . & . & . & . & $\mathrm{Kn}$ & $\mathrm{H}$ & $\mathrm{N}, \mathrm{P}$ \\
\hline+ & + & + & + & + & + & + & . & Ap & $\mathrm{H}$ & $\mathrm{N}, \mathrm{P}$ \\
\hline+ & & + & & + & + & + & . & Ap & $\mathrm{T}$ & $\mathrm{N}, \mathrm{P}$ \\
\hline & . & . & . & . & . & + & . & Ap & $\mathrm{T}$ & $\mathrm{N}, \mathrm{P}$ \\
\hline . & + & . & . & . & . & . & . & $\mathrm{Ar}$ & $\mathrm{T}$ & $\mathrm{N}, \mathrm{P}$ \\
\hline 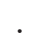 & + & . & . & . & . & . & . & $\mathrm{Sp}$ & $\mathrm{H}$ & $\mathrm{P}$ \\
\hline+ & + & . & . & . & . & . & . & $\mathrm{D}$ & $\mathrm{N}$ & $\mathrm{P}$ \\
\hline . & . & . & . & . & . & + & . & $\mathrm{D}$ & $\mathrm{G}$ & N,P \\
\hline . & + & + & . & . & . & . & . & $\mathrm{Sp}$ & $\mathrm{H}$ & . \\
\hline+ & + & + & . & + & + & + & . & $\mathrm{Ar}$ & $\mathrm{T}$ & $\mathrm{N}, \mathrm{P}$ \\
\hline+ & . & . & . & . & . & . & . & $\mathrm{Sp}$ & $\mathrm{T}$ & $\mathrm{N}, \mathrm{P}$ \\
\hline . & . & . & . & . & . & + & . & $\mathrm{D}$ & $\mathrm{G}$ & $\mathrm{N}, \mathrm{P}$ \\
\hline & . & . & + & . & . & . & . & $\mathrm{Ar}$ & $\mathrm{H}$ & $\mathrm{N}, \mathrm{P}$ \\
\hline 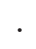 & . & . & . & . & . & + & . & $\mathrm{Ef}$ & $\mathrm{H}$ & $\mathrm{N}, \mathrm{P}$ \\
\hline+ & + & + & + & . & . & + & . & Ap & $\mathrm{H}$ & $\mathrm{N}, \mathrm{P}$ \\
\hline+ & . & . & $\cdot$ & . & . & . & . & cul & $\mathrm{H}$ & $\mathrm{N}, \mathrm{P}$ \\
\hline & . & . & . & . & . & + & . & Ap & $\mathrm{H}$ & $\mathrm{N}, \mathrm{P}$ \\
\hline 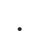 & . & . & . & . & . & + & . & Ap & $\mathrm{G}$ & $\mathrm{N}, \mathrm{P}$ \\
\hline+ & + & + & + & + & + & . & . & Df & $\mathrm{H}$ & N,P \\
\hline+ & + & + & + & . & . & . & . & $\mathrm{Kn}$ & $\mathrm{T}$ & $\mathrm{N}, \mathrm{P}$ \\
\hline+ & . & . & $\cdot$ & . & . & . & . & $\mathrm{Kn}$ & $\mathrm{M}$ & $\mathrm{N}, \mathrm{P}$ \\
\hline+ & + & . & + & . & . & . & . & $\mathrm{D}$ & $\mathrm{Ch}$ & $\mathrm{P}$ \\
\hline+ & . & . & . & . & . & . & . & $\mathrm{Ar}$ & $\mathrm{T}$ & $\mathrm{P}$ \\
\hline+ & . & + & . & + & + & + & . & $\mathrm{Ar}$ & $\mathrm{T}$ & $\mathrm{P}$ \\
\hline+ & . & . & + & + & . & . & . & $\mathrm{D}$ & $\mathrm{T}$ & $\mathrm{P}$ \\
\hline+ & + & + & . & + & . & . & $\cdot$ & $\mathrm{D}$ & $\mathrm{N}$ & . \\
\hline+ & . & + & . & + & . & . & . & Ap & $\mathrm{H}$ & $\mathrm{N}, \mathrm{P}$ \\
\hline & . & . & . & . & . & + & . & $\mathrm{D}$ & $\mathrm{T}$ & $\mathrm{P}$ \\
\hline . & . & . & + & . & + & + & . & $\mathrm{D}$ & $\mathrm{N}$ & $\mathrm{P}$ \\
\hline+ & . & . & . & . & . & . & · & Ap & $\mathrm{H}$ & . \\
\hline . & . & . & . & . & . & + & . & $\mathrm{D}$ & $\mathrm{H}$ & $\mathrm{N}, \mathrm{P}$ \\
\hline+ & . & . & 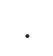 & . & . & . & · & $\mathrm{D}$ & $\mathrm{H}$ & $\mathrm{N}, \mathrm{P}$ \\
\hline+ & . & + & 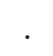 & . & . & + & · & $\mathrm{Kn}$ & $\mathrm{G}$ & . \\
\hline+ & . & . & . & . & . & . & . & $\mathrm{D}$ & $\mathrm{N}$ & . \\
\hline+ & . & . & . & . & $\cdot$ & . & . & $\mathrm{Sp}$ & $\mathrm{M}$ & $\mathrm{P}$ \\
\hline+ & $\cdot$ & $\cdot$ & . & . & . & . & & Ap & $\mathrm{H}$ & $\mathrm{N}, \mathrm{P}$ \\
\hline+ & + & + & $\cdot$ & + & + & $\cdot$ & . & Ap & $\mathrm{H}$ & $\mathrm{N}, \mathrm{P}$ \\
\hline+ & + & + & + & + & + & + & . & Ap & $\mathrm{H}$ & $\mathrm{N}, \mathrm{P}$ \\
\hline
\end{tabular}


Plantago major L. s. str.

Plantago media L.

Pinus sylvestris L.

Poa annua L.

Poa nemoralis L.

Poa pratensis L. s. str.

Poa trivialis L.

Populus alba L.

Populus nigra L.

Populus tremula L.

Polygonum aviculare L.

Polygonum persicaria L.

Portulaca oleracea L.

Potentilla anserina L.

Potentilla argentea $\mathrm{L}$. s. str.

Potentilla reptans $\mathrm{L}$.

Potentilla supina L.

Primula veris $\mathrm{L}$.

Prunella vulgaris $\mathrm{L}$.

Prunus domestica L.

Pteridium aquilinum (L.)Kuhn.

Pulsatilla vulgaris Mill.

Pyrus communis L.

Quercus robur L.

Ranunculus acris L. s. str.

Ranunculus repens $\mathrm{L}$.

Raphanus raphanistrum $\mathrm{L}$.

Reynoutria japonica Houtt.

Rhus typhina L.

Robinia pseudoacacia L.

Rosa rubiginosa $\mathrm{L}$.

Rosa sp. L.

Rubus caesius L.

Rudbeckia hirta L.

Rudbeckia laciniata L.

Rumex acetosa $\mathrm{L}$.

Rumex acetosella $\mathrm{L}$.

Rumex crispus L.

Rumex obtusifolius L.

Ruta graveolens $\mathrm{L}$.

Sagina procumbens L.

Salvia splendens Sello

Salvia verticillata $\mathrm{L}$.

Sambucus nigra $\mathrm{L}$.

Salix ceprea L.

Saponaria officinalis L.

Scleranthus annuus L.

Scrophularia nodosa $\mathrm{L}$.

Secale cereale $\mathrm{L}$.

\begin{tabular}{|c|c|c|c|c|c|c|c|c|c|c|}
\hline+ & + & + & + & + & + & + & $\cdot$ & $\mathrm{Ap}$ & $\mathrm{H}$ & $\mathrm{N}, \mathrm{P}$ \\
\hline & . & + & . & . & . & . & . & Ap & $\mathrm{H}$ & $\mathrm{N}, \mathrm{P}$ \\
\hline+ & . & . & . & . & . & . & . & $\mathrm{Sp}$ & $\mathrm{M}$ & $\mathrm{P}$ \\
\hline+ & + & + & + & + & + & . & . & Ap & $\mathrm{H}$ & . \\
\hline+ & + & + & + & + & + & . & . & $\mathrm{Sp}$ & $\mathrm{H}$ &. \\
\hline+ & + & + & . & . & . & . & . & Ap & $\mathrm{H}$ & . \\
\hline+ & . & . & . & . & . & . & . & Ap & $\mathrm{H}$ &. \\
\hline$\cdot$ & + & . & + & . & . & . & . & Ap & $\mathrm{M}$ & . \\
\hline+ & . & . & . & . & . & . & . & Ap & $\mathrm{M}$ & . \\
\hline+ & . & . & . & . & . & + & . & Ap & $\mathrm{M}$ & . \\
\hline+ & + & + & + & + & + & + & . & Ap & $\mathrm{T}$ & $\mathrm{N}, \mathrm{P}$ \\
\hline+ & . & + & . & . & . & . & . & Ap & $\mathrm{T}$ & $\mathrm{N}, \mathrm{P}$ \\
\hline . & . & . & . & . & + & . & . & $\mathrm{Kn}$ & $\mathrm{T}$ & $\mathrm{P}$ \\
\hline+ & + & + & . & + & . & . & . & Ap & $\mathrm{H}$ & $\mathrm{P}$ \\
\hline+ & . & . & . & . & + & . & . & Ap & $\mathrm{H}$ & $\mathrm{P}$ \\
\hline+ & + & + & . & + & . & + & . & Ap & $\mathrm{H}$ & $\mathrm{P}$ \\
\hline+ & . & . & . & . & . & . & . & Ap & $\mathrm{T}$ & $\mathrm{P}$ \\
\hline . & . & + & . & . & . & . & + & $\mathrm{Sp}$ & $\mathrm{H}$ & $\mathrm{N}, \mathrm{P}$ \\
\hline+ & + & + & . & + & . & . & . & Ap & $\mathrm{H}$ & $\mathrm{N}, \mathrm{P}$ \\
\hline+ & . & . & . & . & . & . & . & $\mathrm{D}$ & $\mathrm{M}$ & $\mathrm{N}, \mathrm{P}$ \\
\hline . & + & . & . & . & . & . & . & $\mathrm{Sp}$ & $\mathrm{G}$ & . \\
\hline+ & . & . & . & + & . & + & + & $\mathrm{Sp}$ & $\mathrm{H}$ & $\mathrm{P}$ \\
\hline . & . & . & . & + & . & . & . & Ap & $\mathrm{M}$ & $\mathrm{N}, \mathrm{P}$ \\
\hline . & + & . & . & . & . & + & . & $\mathrm{Sp}$ & $\mathrm{M}$ & $\mathrm{P}$ \\
\hline+ & + & + & . & & . & . & . & Ap & $\mathrm{H}$ & $\mathrm{P}$ \\
\hline+ & + & + & . & . & . & . & . & Ap & $\mathrm{H}$ & $\mathrm{P}$ \\
\hline . & . & . & . & . & . & + & . & $\mathrm{Ar}$ & $\mathrm{t}$ & $\mathrm{N}, \mathrm{P}$ \\
\hline+ & + & . & + & . & . & . & . & Ken & $\mathrm{G}$ & $\mathrm{P}$ \\
\hline+ & + & . & . & . & . & . & . & $\mathrm{D}$ & $\mathrm{N}$ & $\mathrm{P}$ \\
\hline+ & . & . & + & . & + & + & . & $\mathrm{Kn}$ & $\mathrm{M}$ & $\mathrm{N}, \mathrm{P}$ \\
\hline+ & . & . & . & . & . & + & . & $\mathrm{Kn}$ & $\mathrm{N}$ & $\mathrm{P}$ \\
\hline . & + &.+ & . & . & . & . & . & $\mathrm{D}$ & $\mathrm{N}$ & $\mathrm{P}$ \\
\hline+ & + & + & + & + & . & . & . & Ap & $\mathrm{N}$ & $\mathrm{N}, \mathrm{P}$ \\
\hline+ & + & + & + & + & . & + & . & $\mathrm{D}$ & $\mathrm{H}$ & N,P \\
\hline . & . & . & . & . & . & + & . & $\mathrm{Kn}$ & $\mathrm{H}$ & $\mathrm{N}, \mathrm{P}$ \\
\hline$\cdot$ & . & & & + & + & + & . & Ap & $\mathrm{H}$ & $\mathrm{P}$ \\
\hline+ & . & + & . & . & . & + & . & Ap & $\mathrm{G}$ & $\mathrm{P}$ \\
\hline+ & . & + & + & . & . & . & . & Ap & $\mathrm{H}$ & $\mathrm{P}$ \\
\hline . & + & + & . & . & + & & . & Ap & $\mathrm{H}$ & $\mathrm{P}$ \\
\hline+ & . & . & . & . & . & + & . & $\mathrm{D}$ & $\mathrm{H}$ & $\mathrm{N}, \mathrm{P}$ \\
\hline+ & + & + & + & . & . & . & . & Ap & $\mathrm{H}$ & . \\
\hline . & + & . & . & . & . & . & . & $\mathrm{Kn}$ & $\mathrm{T}$ & $\mathrm{N}, \mathrm{P}$ \\
\hline . & . & . & . & . & . & + & . & Ap & $\mathrm{H}$ & $\mathrm{N}, \mathrm{P}$ \\
\hline . & + & + & + & . & . & + & . & Ap & $\mathrm{N}$ & $\mathrm{P}$ \\
\hline+ & + & + & . & . & . & + & . & Ap & $\mathrm{N}$ & $\mathrm{N}, \mathrm{P}$ \\
\hline+ & + & + & + & + & + & + & . & $\mathrm{Ar}$ & $\mathrm{H}$ & $\mathrm{N}, \mathrm{P}$ \\
\hline & . & . & . & . & + & . & . & Ap & $\mathrm{H}$ & . \\
\hline & + & . & . & . & . & . & . & Ap & $\mathrm{H}$ & $\mathrm{N}, \mathrm{P}$ \\
\hline+ & . & . & . & . & . & . & . & $\mathrm{Ar}$ & $\mathrm{T}$ & \\
\hline
\end{tabular}


Sedum arce $\mathrm{L}$.

Sedum album $\mathrm{L}$.

Sedum maximum (L.)Hoffm.

Sedum hispanicum $\mathrm{L}$.

Sedum spectabile Boreau

Senecio vulgaris $\mathrm{L}$.

Setaria pumila (Poir.) Roem. \& Schult.

Setaria viridis (L.) P. Beauv.

Silene vulgaris (Moench) Garcke

Sinapis arvensis L.

Sisymbrium officinalis L. Scop.

Sisymbrium loeselii L.

Solidago gigantea Aiton

Solidago hybrida hort

Solidago canadensis L.

Sonchus arvensis L.

Sonchus asper (L.) Hill

Sorbus aucuparia $\mathrm{L}$.

Sorbus intermedia (Ehrh.)Pers.

Spergula arvensis L.

Spiraea japonica L.

Spiraea xvanhouttei (Briot)Zabel

Stachys byzantina K. Koch

Stachys sylvatica $\mathrm{L}$.

Stellaria graminea L.

Stellaria media (L.) Vill.

Symphoricarpos albus (L.) S. F. Blake

Symphytum officinale L.

Syringa vulgaris $\mathrm{L}$.

Tagetes patula $\mathrm{L}$.

Tanacetum parthenium (L.) Sch. Bip.

Tanacetum vulgare $\mathrm{L}$.

Taraxacum officinale F.H. Wigg

Thalictrum minus L.

Thlapsi arvense $\mathrm{L}$.

Tilia cordata Mill.

Thuja occidentalis L.

Thymus serphyllum L.

Tragopogon pratensis L. s. str.

Trifolium arvense $\mathrm{L}$.

Trifolium campestre Schreb.

Trifolium dubium Sibth.

Trifolium hybridum L.

Trifolium medium L.

Trifolium pratense $\mathrm{L}$.

Trifolium repens $\mathrm{L}$.

Tussilago farfara $\mathrm{L}$.

Ulmus laevis Pall.

Urtica dioica $\mathrm{L}$.

\begin{tabular}{|c|c|c|c|c|c|c|c|c|c|c|}
\hline+ & + & . & . & + & $\cdot$ & + & . & Ap & $\mathrm{C}$ & N,P \\
\hline+ & . & + & . & . & . & + & . & $\mathrm{Kn}$ & $\mathrm{H}$ & N,P \\
\hline+ & + & & + & . & . & + & . & $\mathrm{D}$ & $\mathrm{H}$ & N,P \\
\hline . & . & . & . & . & . & + & . & $\mathrm{D}$ & $\mathrm{H}$ & $\mathrm{N}, \mathrm{P}$ \\
\hline+ & . & + & . & . & . & + & . & $\mathrm{D}$ & $\mathrm{H}$ & N,P \\
\hline+ & + & + & + & + & + & + & . & $\mathrm{Ar}$ & $\mathrm{T}$ & . \\
\hline . & + & + & + & + & . & . & . & $\mathrm{Ar}$ & $\mathrm{T}$ & . \\
\hline+ & + & + & + & + & + & . & . & $\mathrm{Ar}$ & $\mathrm{T}$ & . \\
\hline+ & . & + & . & . & + & + & . & Ap & $\mathrm{H}$ & $\mathrm{N}, \mathrm{P}$ \\
\hline . & . & + & . & . & . & . & . & $\mathrm{Ar}$ & $\mathrm{T}$ & N,P \\
\hline+ & + & + & + & + & . & . & . & $\mathrm{Ar}$ & $\mathrm{T}$ & $\mathrm{N}, \mathrm{P}$ \\
\hline+ & . & + & + & . & + & . & . & $\mathrm{Ar}$ & $\mathrm{T}$ & N,P \\
\hline+ & . & . & . & . & . & . & . & $\mathrm{Kn}$ & $\mathrm{G}$ & N,P \\
\hline+ & + & . & + & . & . & + & . & cul & $\mathrm{G}$ & $\mathrm{N}, \mathrm{P}$ \\
\hline+ & + & . & + & + & + & . & . & $\mathrm{Kn}$ & $\mathrm{G}$ & N,P \\
\hline+ & + & + & + & + & + & . & . & Ap & $\mathrm{H}$ & $\mathrm{N}, \mathrm{P}$ \\
\hline+ & . & . & + & . & + & . & . & $\mathrm{Ar}$ & $\mathrm{T}$ & N,P \\
\hline+ & + & + & + & . & . & . & . & Ap & $\mathrm{M}$ & $\mathrm{N}, \mathrm{P}$ \\
\hline+ & + & + & . & . & . & . & . & $\mathrm{D}$ & $\mathrm{M}$ & N,P \\
\hline+ & . & + & . & . & . & + & . & $\mathrm{Ar}$ & $\mathrm{T}$ & $\mathrm{P}$ \\
\hline+ & & . & . & . & . & . & . & $\mathrm{D}$ & $\mathrm{N}$ & $\mathrm{P}$ \\
\hline . & + & . & . & . & . & . & . & $\mathrm{D}$ & $\mathrm{N}$ & $\mathrm{P}$ \\
\hline+ & . & . & . & . & . & + & . & $\mathrm{D}$ & $\mathrm{H}$ & $\mathrm{N}, \mathrm{P}$ \\
\hline . & . & . & . & + & . & . & . & $\mathrm{Sp}$ & $\mathrm{H}$ & $\mathrm{N}, \mathrm{P}$ \\
\hline+ & . & . & . & . & . & . & . & Ap & $\mathrm{H}$ & $\mathrm{N}, \mathrm{P}$ \\
\hline+ & . & . & . & + & . & . & . & Ap & $\mathrm{T}$ & N,P \\
\hline . & + & + & + & + & + & + & . & $\mathrm{Kn}$ & $\mathrm{N}$ & $\mathrm{N}, \mathrm{P}$ \\
\hline+ & + & + & + & + & + & . & . & $\mathrm{Sp}$ & $\mathrm{H}$ & N,P \\
\hline+ . & + & + & + & + & . & + & . & $\mathrm{Kn}$ & $\mathrm{N}$ & $\mathrm{P}$ \\
\hline+ & . & + & . & . & + & + & . & cul & $\mathrm{T}$ & $\mathrm{P}$ \\
\hline+ & . & . & + & . & . & . & . & $\mathrm{Kn}$ & $\mathrm{H}$ & $\mathrm{P}$ \\
\hline+ & + & + & + & + & + & + & . & Ap & $\mathrm{H}$ & $\mathrm{P}$ \\
\hline+ & + & + & + & + & + & + & . & Ap & $\mathrm{H}$ & N,P \\
\hline$\cdot$ & $\cdot$ & $\cdot$ & + & $\cdot$ & $\cdot$ & $\cdot$ & . & $\mathrm{Sp}$ & $\mathrm{H}$ & $\mathrm{P}$ \\
\hline . & . & . & . & . & . & + & . & $\mathrm{Ar}$ & $\mathrm{T}$ & $\mathrm{N}, \mathrm{P}$ \\
\hline+ & + & + & + & + & . & . & . & Ap & $\mathrm{M}$ & $\mathrm{N}, \mathrm{P}$ \\
\hline+ & + & + & + & . & . & . & . & $\mathrm{D}$ & $\mathrm{N}$ & $\mathrm{P}$ \\
\hline+ & + & . & $\cdot$ & . & . & . & . & Ap & $\mathrm{C}$ & $\mathrm{N}, \mathrm{P}$ \\
\hline+ & + & + & . & + & + & . & . & Ap & $\mathrm{T}$ & $\mathrm{N}, \mathrm{P}$ \\
\hline . & . & . & . & + & . & . & . & Ap & $\mathrm{T}$ & $\mathrm{N}, \mathrm{P}$ \\
\hline+ & . & . & . & . & . & . & . & Ap & $\mathrm{T}$ & N,P \\
\hline+ & . & . & $\cdot$ & . & . & . & . & Ap & $\mathrm{T}$ & $\mathrm{N}, \mathrm{P}$ \\
\hline+ & . & + & . & . & . & . & . & Ap & $\mathrm{H}$ & $\mathrm{N}, \mathrm{P}$ \\
\hline+ & + & . & . & . & . & . & . & Ap & $\mathrm{H}$ & $\mathrm{N}, \mathrm{P}$ \\
\hline+ & + & + & . & + & + & + & . & Ap & $\mathrm{H}$ & $\mathrm{N}, \mathrm{P}$ \\
\hline+ & + & + & + & + & + & + & . & Ap & $\mathrm{H}$ & N,P \\
\hline+ & + & + & . & + & + & + & . & Ap & $\mathrm{G}$ & $\mathrm{N}, \mathrm{P}$ \\
\hline$\cdot$ & . & $\cdot$ & + & $\cdot$ & $\cdot$ & . & - & Ap & $\mathrm{M}$ & $\mathrm{P}$ \\
\hline+ & + & + & + & + & + & . & . & Ap & $\mathrm{H}$ & $\mathrm{P}$ \\
\hline
\end{tabular}




\begin{tabular}{|c|c|c|c|c|c|c|c|c|c|c|c|}
\hline Verbascum phoeniceum L. & . & & & & & + & + & . & Ap & $\mathrm{H}$ & $\mathrm{P}$ \\
\hline Verbascum nigrum L. & + & + & + & + & + & . & + & . & Ap & $\mathrm{H}$ & $\mathrm{P}$ \\
\hline Verbascum densiflorum Bertol. & . & . & . & . & . & . & + & . & $\mathrm{Sp}$ & $\mathrm{H}$ & $\mathrm{P}$ \\
\hline Veronica arvensis L. & + & + & + & . & + & + & . & . & Ap & $\mathrm{T}$ & $\mathrm{N}, \mathrm{P}$ \\
\hline Veronica chamaedrys L. s.str. & + & + & + & . & . & . & . & . & Ap & $\mathrm{H}$ & $\mathrm{N}, \mathrm{P}$ \\
\hline Veronica hederifolia L. s. str. & + & . & . & . & . & . & . & . & $\mathrm{Kn}$ & $\mathrm{T}$ & $\mathrm{N}, \mathrm{P}$ \\
\hline Veronica polita Fr. & + & + & + & + & . & . & . & . & $\mathrm{Ar}$ & $\mathrm{T}$ & $\mathrm{N}, \mathrm{P}$ \\
\hline Viburnum lantana $\mathrm{L}$. & + & . & . & . & . & . & . & . & $\mathrm{Kn}$ & $\mathrm{N}$ & $\mathrm{P}$ \\
\hline Viburnum opulus $\mathrm{L}$. & . & . & . & + & . & . & . & + & $\mathrm{Sp}$ & $\mathrm{N}$ & $\mathrm{P}$ \\
\hline Vicia cracca $\mathrm{L}$. & + & + & + & + & + & + & + & . & Ap & G & $\mathrm{N}, \mathrm{P}$ \\
\hline Vicia hirsuta (L.) Gray & . & . & . & . & + & . & . & . & $\mathrm{Ar}$ & $\mathrm{T}$ & $\mathrm{N}, \mathrm{P}$ \\
\hline Vicia sepium $\mathrm{L}$. & + & + & + & . & + & + & . & . & $\mathrm{Sp}$ & $\mathrm{G}$ & $\mathrm{N}, \mathrm{P}$ \\
\hline Vicia tetrasperma (L.) Schreb. & + & + & . & . & . & . & . & . & $\mathrm{Ar}$ & $\mathrm{T}$ & $\mathrm{N}, \mathrm{P}$ \\
\hline Vinca minor $\mathrm{L}$. & + & + & . & + & . & . & + & + & $\mathrm{Sp}$ & $\mathrm{Ch}$ & $\mathrm{N}, \mathrm{P}$ \\
\hline Viola xwittrockiana Hort. & + & + & . & + & . & . & + & . & $\mathrm{D}$ & $\mathrm{T}$ & $\mathrm{N}, \mathrm{P}$ \\
\hline Viola arvensis Murray & + & + & . & . & . & . & + & . & $\mathrm{Ar}$ & $\mathrm{T}$ & $\mathrm{N}, \mathrm{P}$ \\
\hline Viola odorata $\mathrm{L}$. & + & + & + & . & + & . & + & . & $\mathrm{Kn}$ & $\mathrm{H}$ & $\mathrm{N}, \mathrm{P}$ \\
\hline Viscaria vulgaris Röhl. & + & . & . & . & . & . & . & . & $\mathrm{Sp}$ & $\mathrm{H}$ & $\mathrm{N}, \mathrm{P}$ \\
\hline Yucca filamentosa $\mathrm{L}$. & + & . & . & . & . & . & . & . & $\mathrm{D}$ & $\mathrm{H}$ & $\mathrm{P}$ \\
\hline
\end{tabular}

Explanation: cemetery M - Majdanek, L - Lipowa, U - Unicka, K - Kalina, Łę - Łęczna, Łu - Łuszczów, O - Ostrówek, PS (Protected species), GHs (Geographical-historical status): Ap - apophytes, Ar - archeophytes, Sp - spontaneophytes, Kn - kenophytes, D - diaphytes, cul - cultivated, $\mathrm{N}$ - indefinite, LF (Life forms): $\mathrm{M}$ - megaphanerophytes, $\mathrm{N}$ - nanophanerophytes, $\mathrm{Ch}$ - chamaephytes, $\mathrm{G}$ - geophytes, $\mathrm{H}$ - hemicryptophytes, $\mathrm{T}$ - therophytes, UF (Usage form): $\mathrm{N}$ - nectariferous, $\mathrm{P}$ - polleniferous.

\section{The percentage of life forms}

The percentage of species belonging to different life forms is similar for all of the cemeteries analysed (Table 2). The highest percentage of hemicryptophytes was observed in Ostrówek (50.7 \%), while the lowest one in the Kalina cemetery $(40.3 \%)$. In the case of therophytes, the highest level of species diversity was observed in the urban cemetery on Unicka Street (32.6\%), while the lowest - in Ostrówek. The average percent- age of geophytes in the flora of the analysed cemeteries was $12.1 \%$; it was the highest in Łuszczów (16.4\%) and the lowest in Majdanek (8.4\%). There was a low percentage of chamaephytes (from $5.1 \%$ to $4.4 \%$ ), nanophanerophytes $(6.2 \%$ to $2.7 \%)$, and megaphanerophytes $(9.6 \%-1.8 \%)$. In the case of the last two forms, in terms of percentage, the cemetery on Lipowa Street differed from the others and was characterised by the highest level of deliberately introduced diversity of the forest stand.

Table 3

Number of species and the proportions of different life forms in the flora of the examined cemeteries in Lublin, Łęczna, Łuszczów, and Ostrówek

\begin{tabular}{|c|c|c|c|c|c|c|c|c|c|c|c|c|c|c|}
\hline \multirow{3}{*}{ Life forms } & \multicolumn{14}{|c|}{ Flora of cemeteries } \\
\hline & \multicolumn{2}{|c|}{ Majdanek } & \multicolumn{2}{|c|}{ Lipowa } & \multicolumn{2}{|c|}{ Unicka } & \multicolumn{2}{|c|}{ Kalina } & \multicolumn{2}{|c|}{ Łęczna } & \multicolumn{2}{|c|}{ Łuszczów } & \multicolumn{2}{|c|}{ Ostrówek } \\
\hline & $\begin{array}{c}\text { number } \\
\text { of } \\
\text { species }\end{array}$ & $\%$ & $\begin{array}{c}\text { number } \\
\text { of } \\
\text { species }\end{array}$ & $\%$ & $\begin{array}{l}\text { number } \\
\text { of } \\
\text { species }\end{array}$ & $\%$ & $\begin{array}{c}\text { number } \\
\text { of } \\
\text { species }\end{array}$ & $\%$ & $\begin{array}{c}\text { number } \\
\text { of } \\
\text { species }\end{array}$ & $\%$ & $\begin{array}{c}\text { number } \\
\text { of } \\
\text { species }\end{array}$ & $\%$ & $\begin{array}{l}\text { number } \\
\text { of species } \\
\text { taksonów }\end{array}$ & $\%$ \\
\hline hemicryptophytes & 133 & 48.6 & 81 & 46.0 & 83 & 43.0 & 50 & 40.3 & 62 & 42.5 & 45 & 41.0 & 74 & 50.7 \\
\hline therophytes & 80 & 29.2 & 39 & 22.2 & 63 & 32.6 & 37 & 29.8 & 47 & 32.2 & 39 & 35.4 & 34 & 23.3 \\
\hline geophytes & 23 & 8.4 & 19 & 10.8 & 20 & 10.4 & 16 & 13.0 & 19 & 13.0 & 18 & 16.4 & 19 & 13.0 \\
\hline chamaephytes & 12 & 4.4 & 9 & 5.1 & 7 & 3.6 & 6 & 4.8 & 5 & 3.4 & 3 & 2.7 & 7 & 4.8 \\
\hline nanophanerophytes & 13 & 4.7 & 11 & 6.3 & 8 & 4.2 & 6 & 4.8 & 4 & 2.7 & 3 & 2.7 & 6 & 4.1 \\
\hline megaphanerophytes & 13 & 4.7 & 17 & 9.6 & 12 & 6.2 & 9 & 7.3 & 9 & 6.2 & 2 & 1.8 & 6 & 4.1 \\
\hline total & 274 & 100 & 176 & 100 & 193 & 100 & 124 & 100 & 146 & 100 & 110 & 100 & 146 & 100 \\
\hline
\end{tabular}




\section{The origin of plants observed in the cemeteries}

Among the spontaneously occuring plants, the cemeteries in question are dominated by apophytes, the percentage of which varies between 52.3\% in Lublin on Lipowa and Unicka Streets to $44.5 \%$ in Ostrówek (Table 3). Apophytes originate from forest, lawn, and meadow habitats. In shaded locations in the cemeteries, one can observe: Mycelis muralis, Lapsana communis, Aegopodium podagraria, which originate in forests. Due to a high level of insolation as well as large amounts of sand used for construction work and water shortages in all the cemeteries, except the one in Lipowa Street, well-represented are groups of species of psammophilic vegetation: Hieracium pilosella, Sedum acre, Rumex acetosella, Carex hirta, forest edge vegetations: Coronilla varia, Galium verum, Centaurea jacea, and meadow species: Pimpinella major, Plantago lanceolata, Ranunculus acer, $R$. repens. Species originating in meadow communities are dominant, except for the
Kalina cemetery (35.5\%); their percentage ranges from $43.5 \%$ (Lipowa) to $53.3 \%$ (Łęczna). In the older forested cemeteries, above $30 \%$ are species originating in forest communities - Lipowa (30 species, i.e. 32.6\%), Kalina (21 species, $33.9 \%$ ). Sunlit locations were dominated by lawn species: Majdanek (31.3\%), Ostrówek (35.4\%), and Łuszczów (35.9\%). Dominant among antropophytes are archeophytes, although their percentage ranges from $26.3 \%$ in Łuszczów to $13.1 \%$ on Lipowa Street. A similar percentage characterises diaphytes and kenophytes. The percentage of diaphytes in the cemetery flora varies from $16.5 \%$ to $7.2 \%$, while that of kenophytes from $15.1 \%$ in Ostrówek to $8.2 \%$ in Łęczna. In the case of spontaneophytes, the percentage of which ranges between $8.8 \%$ and $5.2 \%$, quite significant is only $2.7 \%$ of them in the Łuszczów cemetery. Among spontaneous species encountered in the cemeteries, one can observe a group of ornamental plants, plantation "runaways": Convallaria majalis, Cosmos bipinnatus, Hemerocallis fulva, Rudbeckia hirta, Lysimachia punctata.

Table 4

Number of species and the proportions of geographical-historical status groups in the flora of the examined cemeteries in Lublin, Łęczna, Łuszczów, and Ostrówek

\begin{tabular}{|c|c|c|c|c|c|c|c|c|c|c|c|c|c|c|}
\hline \multirow{3}{*}{$\begin{array}{l}\text { Geographical- } \\
\text { historical } \\
\text { groups }\end{array}$} & \multicolumn{14}{|c|}{ Flora of cemeteries } \\
\hline & \multicolumn{2}{|c|}{ Majdanek } & \multicolumn{2}{|c|}{ Lipowa } & \multicolumn{2}{|c|}{ Unicka } & \multicolumn{2}{|c|}{ Kalina } & \multicolumn{2}{|c|}{ Łęczna } & \multicolumn{2}{|c|}{ Łuszczów } & \multicolumn{2}{|c|}{ Ostrówek } \\
\hline & $\begin{array}{l}\text { number } \\
\text { of } \\
\text { species }\end{array}$ & $\%$ & $\begin{array}{c}\text { number } \\
\text { of } \\
\text { species }\end{array}$ & $\%$ & $\begin{array}{l}\text { number } \\
\text { of } \\
\text { species }\end{array}$ & $\%$ & $\begin{array}{c}\text { number } \\
\text { of } \\
\text { species }\end{array}$ & $\%$ & $\begin{array}{c}\text { number } \\
\text { of } \\
\text { species }\end{array}$ & $\%$ & $\begin{array}{c}\text { number } \\
\text { of } \\
\text { species }\end{array}$ & $\%$ & $\begin{array}{c}\text { number } \\
\text { of } \\
\text { species }\end{array}$ & $\%$ \\
\hline apophytes & 128 & 46.7 & 92 & 52.3 & 101 & 52.3 & 62 & 50.0 & 75 & 51.4 & 56 & 51.0 & 65.0 & 44.5 \\
\hline archeophytes & 46 & 16.8 & 23 & 13.1 & 37 & 19.2 & 25 & 20.3 & 31 & 21.2 & 29 & 26.3 & 21 & 14.4 \\
\hline kenophytes & 35 & 12.8 & 25 & 14.2 & 21 & 10.9 & 17 & 13.7 & 12 & 8.2 & 14 & 12.7 & 22 & 15.1 \\
\hline spontaneophytes & 24 & 8.8 & 14 & 7.9 & 10 & 5.2 & 8 & 6.4 & 10 & 6.8 & 3 & 2.7 & 10 & 6.8 \\
\hline diaphytes & 39 & 14.2 & 21 & 11.9 & 24 & 12.4 & 9 & 7.2 & 16 & 11.0 & 8 & 7.3 & 24 & 16.5 \\
\hline indefinite & 2 & 0.7 & & 0.6 & 0 & 0 & 3 & 2.4 & 2 & 1.4 & 0 & 0 & 4 & 2.7 \\
\hline total & 274 & 100 & 176 & 100 & 193 & 100 & 124 & 100 & 146 & 100 & 110 & 100 & 146 & 100 \\
\hline
\end{tabular}

\section{Floristic diversity in terms of the degree of anthropopression}

The analysis of species composition of the cemeteries in question allows us to confirm the presence of 33 recurring species: Achillea millefolium, Aegopodium podagraria, Artemisia vulgaris, Ballota nigra, Capsella bursa-pastoris, Chamomilla suaveolens, Chelidonium majus, Chenopodium album, Convallaria majalis, Convolvulus arvensis, Conyza canadensis, Elymus repens, Erigeron annuus, Fallopia convolvulus, Festuca ovina, F. rubra, Galinsoga parviflora, Geranium pusillum, Hemerocallis fulva, Impatiens parviflora, Lolium perenne, Melandrium album, Plantago lanceolata, Plantago major, Polygonum aviculare,
Rudbeckia hirta, Saponaria officinalis, Syringa vulgaris, Tanacetum vulgare, Taraxacum officinale, Trifolium repens, Vicia cracca.

In the urban cemeteries in Lublin, where 340 species were found, 36 were present at all the sites: Acer pseudoplatanus, Arctium sp., Calamagrostis epigejos, Campanula trachelium, Carex hirta, Cosmos bipinnatus, Descurainia sophia, Dianthus barbatus, Epilobium hirsutum, Erisimum cheiranthoides, Erodium cicutarium, Euphorbia peplus, Geranium pratensis, Geum urbanum, Lactuca serriola, Lamium album, Lapsana communis, Linaria vulgaris, Lysimachia punctata, Oenothera biennis, Oxalis corniculatum, O. dillenii, Poа апnиа, P. nemoralis, Rubus sp., 
Sagina procumbens, Sedum spectabile, Setaria viridis, Sherardia arvensis, Sisymbrium officinalis, Sonchus arvensis, Sorbus aucuparia, Thuja sp., Tilia cordata, Urtica dioica, Verbascum nigrum.

In the rural cemeteries (196 species), 34 species recurred: Allium vineale, Apera spica-venti, Cichorium intybus, Cirsium arvense, Coronilla varia, Echinochloa crus-galli, Equisetum arvense, Eryngium campestre, Festuca pratensis, Fumaria schleicheri, Glechoma hederacea, Helianthus rigidus, Heracleum sphondylium, Hypericum perforatum, Iris germanica, Leucanthemum vulgare, Lupinus polyphyllus, Medicago lupulina, Melilotus albus, Myosotis arvensis, Philadelphus coronarius, Polygonum persicaria, Robinia pseudoacacia, Rumex acetosa, R. acetosella, Sedum acre, Senecio vulgaris, Silene vulgaris, Solidago ssp., Symphoricarpos albus, Tagetes patula, Trifolium pratense, Tussilago farfara, Verbascum phoeniceum.

The percentage of honey species (polleniferous and nectariferous) and protected species

Cemeteries, often called the gardens of memory, surrounded by urban and suburban development, have been for ages "islands" of green. However, falling trees, which are potentially dangerous, produce waste and may damage tombstones; the lack of deliberate plantings, the presence of large impenetrable areas (cobblestones enclosing the tombs) are also the reasons why cemeteries are losing their high rank as green areas (S o b c zak et al. 2004). Nevertheless, the analysis of the level of diversity of spontaneously occuring plants showed quite significant species diversification. The majority of them, 299 species, are invaluable to insects (polleniferous andnectariferous) (Table 2). The presence of spontaneous plants in cemeteries contributes to the food base for animals. What is interesting is the fact that all of the recorded species are equally visited by insects. The following are among the species that are visited most frequently: Ballota nigra, Bunias orientalis, Campanula sp., Centaurea sp., Lamium album, Tilia cordata. There are also 11 protected species among the species observed in the cemeteries.

\section{DISCUSSION}

Cemeteries, as burial grounds, preform funeral, cultural, and aesthetic functions, and due to a high percentage of sown and planted species, they increase floristic diversity. They are considered invaluable green areas which are included in "The Municipal Natural System" (S zulczewska and Kaftan, 1996). They can play the role of a repository for indigenous species, which was confirmed by the research conducted in the cemeteries of Jarocin ( $\mathrm{C} \mathrm{z}$ a r n a , 2004) and Warsaw (G a le ra et al. 1993).

A comparison of the percentage of species found in the cemeteries in large cities of Poznań $(\mathrm{C} \mathrm{z}$ a rn a et al. 2011) and Lublin allows the conclusion that the number of species is similar. In the cemeteries of Poznań, 395 species were found, while in Lublin 340. However, the cemeteries investigated in Poznań are small sites $(0.25-8 \mathrm{ha})$, situated in the city centre and established at the beginning of the $20^{\text {th }}$ century. Therefore, they differ significantly from those in Lublin. Despite the differences, the analysed cemeteries are dominated by persistent forms: hemicryptophytes, chamaephytes, nanophanerophytes, and megaphanerophytes, although their percentage in the cemeteries of Lublin is higher; in Poznan it varies between 32.2 and $41.7 \%$, whereas in Lublin between 46.7 and $52.3 \%$. The list of species considered to occur constantly in cemeteries is similar. The species that recur in Lublin and Poznań are the following: Aegopodium podagraria, Ballota nigra, Convallaria majalis, Hemerocallis fulva, Saponaria officinallis, and Solidago ssp.

The analysis of the cemeteries in Jarocin (C z a r n a , 2004), Ostrów Wielkopolski and its vicinity $(\mathrm{Celka}$ and $\dot{\mathrm{Z} y w i c k a, 2004)}$ as well as Jelenia Góra and its vicinity (C z a r n a et al. 2006) shows that, as in Lublin, the dominant plants are apophytes and persistent species, out of which perennial herbacious plants are represented the best. Many of these cemeteries have valuable and protected plants (Galera et al. 1983). Among the plants spontaneously occuring in cemeteries, there are also indigenous species that grow freely, which is confirmed by the analyses of the flora of the necropolises in the Slovinski National Park (Sobisz and Antkowiak, 2009), Koźm in and Koźminiec (C z a rn a, 2001) as well as Jarocin ( $\mathrm{C} \mathrm{z}$ a $\mathrm{n}$ a et al. 2006). Species present nearly in all cemeteries are the following, for instance: Hedera helix, Syringa vulgaris, Vinca minor, Aquilegia xhybrida, Galanthus nivalis, Hemerocallis fulva, Muscari neglectum, Ornithogalum umbellatum, Polygonatum multiflorum, Primula elatior, Primula veris, and Viola odorata. However, the research conducted in other cemeteries, for instance in Jelenia Góra and its vicinity ( $\mathrm{Cz}$ arn a et al. 2006), also found other species: $A s$ tragalus glycyphyllos, Centaurea mollis, Digitalis purpurea, Erigeron annuus, E. ramosum, Leucanthemum vulgare, Lupinus polyphyllus, and Solidago canadensis. A comparison of species considered to be constant ( $\mathrm{Cz}$ ar n a 2004) with those found in Lublin and its vicinity indicates a small number of common species.

In the case of cemeteries, apart from the advantages due to the abundance of spontaneous species, there is also a danger of spread of invasive species such as: Solidago canadensis, Reynoutria japonica, Erigeron annuus, and Conyza canadensis, which was described in the papers by $\mathrm{Czarna}$ (2004) and Galera (2011). It is also influenced by the introduction of new species due to planting and sowing of ornamental species, transfering new diasporas with nursery material 
and potted plants ( $\mathrm{G}$ ale $\mathrm{ra}$ et al. 1993), maintenance of the surroundings of the graves as well as works associated with the construction and maintenance of tombstones.

\section{CONCLUSIONS}

In the seven analysed necropolises of Lublin and its vicinity, a total number of 382 species was found; the highest number, 276, was found in the largest and youngest cemetery in Majdanek situated on the outskirts of Lublin. The smallest number was found in the smallest cemetery in the village of Łuszczów, 110 species. In all of the cemeteries, herbaceous perennial species - hemicryptophytes $(44.6 \%)$ - were the dominant plants. The highest percentage $(49.7 \%)$ was that of apophytes originating in forest, lawn, and meadow habitats. Their presence was dependent on insolation conditions, number of groups of trees, time of establishment, and diversity of graves (earth graves, stone tombstones). In the older forested cemeteries (Lipowa, Kalina), the dominant species are those originating in forest communities. In insolated locations, the dominant plants were lawn species. All of the cemeteries presented an abundance of antropophytes and most of them were dominated by archeophytes, the percentage of which was approximately $20 \%$; only in the case of the oldest cemetery on Lipowa Street this percentage was smaller and it was $13.1 \%$.

Despite the similar proportions in the presence of species belonging to different life forms as well as the geographical-historical origin of plants, the individual cemeteries differ significantly in terms of species composition. In the analysed cemeteries, only 33 species are shared by urban and rural complexes. Out of 340 species found in the urban cemeteries, only 36 are shared by four of the sites. In the case of the rural cemeteries, out of 196 species, 34 were found in three of the sites investigated.

All of the analysed cemeteries present an abundance of honey plants which constitute $78 \%$ of all species recorded. This shows that these sites can be considered invaluable for animals and contribute to increased biological diversity.

\section{Acknowledgements}

Research supported by activities of Departament of Landscape Architecture, Catholic University of Lublin John Paul II.

\section{Authors' contributions}

The following declarations about authors' contributions about authors' to the research have been made: concept of the study: ET, field work ET, KK, writing: ET, KK.

\section{REFERENCES}

Celka Z., Żywicka J. 2004. Flora naczyniowa wybranych cmentarzy Ostrowa Wielkopolskiego i okolic. / Vascular flora of selected cementeries in OstrówWielkopolski and its surroundings. Rocz. Akademii Rolniczej Poznań 363 Bot 7: 1-31 (in Polish)

Czarna A. 2001. Flora naczyniowa cmentarzy ewangelickich w Koźminie i Koźmińcu (Nizina Wielkopolska). I Vascular flora of the Evangelical cemeteries in Koźmin and Koźminiec (Wielkopolska Lowland). Rocz. Akademii Rolniczej w Poznaniu 334: 27-37 (in Polish)

Czarna A. 2004. Flora naczyniowa cmentarzy na terenie Jarocina. / Vascular flora of the cemeteries in the town of Jarocin. Rocz. Akademii Rolniczej w Poznaniu 363: 33-45 (in Polish)

Czarna A., Nowińska R. 2011. Vascular flora in cementeries of the Roztocze region and surrounding areas (south-east Poland). Acta Agrobot. 64(2): 77-92

Czarna A., Piskorz R., Wyrzykiewicz-Raszews k a M. 2006. Vascular plants on selected catholic cemeteries of Jelenia Góra and its surroundings. Rocznik AR Poznań 378 (10): 69-86

Czarna A., Woźnicka A., Maj M., Morozowska M. 2011. Flora of vascular plants of selected Poznań cemeteries. Acta Agrobot. 64(4): 123-140

Czekalski M. 2001. Wieloletnie ozdobne rośliny zielne stosowane do dekoracji grobów na cmentarzu parafialnym w Lesznie w latach 1998-2000. / Perennial ornamental plants used for decoration of graves in the Catholic parish cemetery in Leszno in 1998-2000. Zeszyty Naukowe Akademii Rolniczej im. H. Kołłątaja w Krakowie 379: 67-73 (in Polish)

Dąbski M., Oleś A. 2006. Analiza dendroliogiczna zabytkowego cmentarza przy ulicy Lipowej w Lublinie. / The dendrology analysis of the historic cemetery on Lipowa street in Lublin. Przyroda i miasto, 8: 434-440 (in Polish)

Dębicz R. 2002. Częstotliwość występowania roślin rosnących na grobach i obok nich na cmentarzach we Wrocławiu. / Frequency of occurrence of plants growing on and around graves in the cemeteries of Wrockaw city. Zesz. Probl. Post. Nauk Roln. 483: 47-53 (in Polish)

Dębicz R. 2003. Częstość stosowania kwiatów ciętych do dekoracji grobów na cmentarzach we Wrocławiu. / The Frequency of Applying of Cut Flowers to Decorate Graves in Cemeteries in Wrocław. Acta Sci. Pol. Hortorum Cultus, 2(2): 25-31(in Polish)

Denisow B., Wrzesień M. 2007. The anthropogenic refuge areas for bee flora in agricultural landscape. Acta Agrobot. 60(1): 147-159

Erhardt W., Götz E., Bödeker N., Seybold S. 2000. Zander Handwörterbuch der Pflanzennamen. Eugen Ulmer, Stuttgart: 990

Galera H., Sudnik-Wójcikowska B., Lisowska M. 1993. Flora cmentarzy lewobrzeżnej Warszawy na tle flory miasta. / Flora of the cemeteries of the left-bank 
part of Warsaw compared to the flora of the whole city. Fragm. Flor. Geobot. 38(1): 237-261 (in Polish)

Jackowiak B. 1990. Antropogeniczne przemiany flory roślin naczyniowych Poznania. Wydawnictwo Naukowe UAM, Poznań: 232 (in Polish)

Jędrzejko K., Walusiak E. 2010. Wieloaspektowość i specyfika kulturowa badań przyrodniczych na cmentarzach - w nawiązaniu do badań florystycznych i fitosocjologicznych na obszarze Podbeskidzia Zachodniokarpackiego (Podgórze Śląskie i Wielickie). / Multi-aspect character and cultural problems of environmental studies at cemeteries - in reference to vegetation analyses and phytosociological studies in the area of Podbeskidzie Zachodniokarpackie (Pogórze Śląskie and Wielickie). Problemy Ekologii 14 (2): 98-105 (in Polish)

Lipiński M. 2010. Pożytki pszczele zapylenie i miododajność roślin. PWRiL, Warszawa, Wydawnictwo Sądecki Bartnik, Stróże: 319 (in Polish)

Mosek B., Miazga S. 2005. Trawy w zbiorowiskach roślinnych nekropolii lubelskich. / Grasses in plant communities of cemeteries in Lublin (research note). Łąkarstwo w Polsce 8: 289-296 (in Polish)

Rutkowski L. 2007. Klucz do oznaczania roślin naczyniowych Polski niżowej. PWN, Warszawa: 814 (in Polish)

Sic iński J.T. 1989. Zieleń łódzkich cmentarzy. / Greenery of the Łódź cemeteries. [In:] R. Olaczek, G. Ojrzanowski (eds.). W obronie zieleni miast. Wyd. Uniwersytetu Łódzkiego, Łódź: 35-41(in Polish)

Siewniak M, Mitkowska A. 1998. Tezaurus sztuki ogrodowej. Oficyna Wydawnicza Rytm. Warszawa: 350 (in Polish)

Sobczak K., Trzaskowska E., Falińska-Król J. 2004. Zieleń wysoka na współczesnych cmentarzach wiejskich Lubelszczyzny. / Tall greenery in the contemporary cemeteries of Lubelszczyzna. [In:] J. Janecki, Z. Borkowski (eds.). Krajobraz i ogród wiejski t.3., Lublin: 121-125 (in Polish)

Sobisz Z., Antkowiak W. 2009. Flora naczyniowa cmentarzy na obszarze Słowińskiego Parku Narodowego. / Vascular flora of cemetaries of Słowiński National Park Area. Słupskie Prace Biologiczne, 6: 127-144 (in Polish)
Szulczewska B., Kaftan J. 1996. Kształtowanie systemu przyrodniczego miasta. IGPiK, Warszawa: 1-72 (in Polish)

Wrzesień M., Denisow B. 2006. The usable taxons in spontaneous flora of railway areas of the central-eastern part of Poland. Acta Agrobot. 59(2): 95-108

Zarzycki K., Trzcińska-Tacik H., Różański W., Szeląg Z., Wołek J. 2002. Ekologiczne liczby wskaźnikowe roślin naczyniowych Polski. / Ecological indicator values of vascular plants of Poland. W. Szafer Institute of Botany Polish Academy of Sciences, Kraków: 183

\section{Spontaniczna flora naczyniowa wybranych cmentarzy Lublina i okolic}

\section{Streszczenie}

Praca przedstawia wyniki badań przeprowadzonych na 7 cmentarzach Lubelszczyzny: 4 z nich to cmentarze wielkomiejskie (Lublin), 1 położony jest w małym miasteczku (Łęczna), 2 obiekty to cmentarze wiejskie (Łuszczów, Ostrówek). Badania miały na celu inwentaryzację występującej spontanicznej flory, opisano ją pod kątem przynależności do grup geograficzno-historycznych, form życiowych i obecności gatunków pożytkowych. Na terenie badań stwierdzono występowanie 382 gatunków, największe zróżnicowanie występowało na cmentarzach miejskich 340 a jedynie 110 na terenach wiejskich. Największe zróżnicowanie gatunkowe stwierdzono na największym i najmłodszym cmentarzu w Lublinie na Majdanku. Na wszystkich cmentarzach dominują formy trwałe hemikryptofity oraz apofity. Bardzo dużą grupę stanowią gatunki pożytkowe aż $78 \%$ wszystkich gatunków spotykanych na cmentarzach. W składzie gatunkowym występuje bardzo duże zróżnicowanie tylko 33 gatunki są wspólne dla wszystkich obiektów. Odnaleziono również 36 gatunków na 340 wspólnych dla cmentarzy miejskich oraz 34 gatunki na 196 dla cmentarzy wiejskich. 
